\title{
An Adaptive Primal-Dual Framework for Nonsmooth Convex Minimization
}

\author{
Quoc Tran-Dinh • Ahmet Alacaoglu • \\ Olivier Fercoq • Volkan Cevher
}

Received: date / Accepted: date

\begin{abstract}
We propose a new self-adaptive, double-loop smoothing algorithm to solve composite, nonsmooth, and constrained convex optimization problems. Our algorithm is based on Nesterov's smoothing technique via general Bregman distance functions. It selfadaptively selects the number of iterations in the inner loop to achieve a desired complexity bound without requiring the accuracy a priori as in variants of Augmented Lagrangian methods (ALM). We prove $\mathcal{O}\left(\frac{1}{k}\right)$-convergence rate on the last iterate of the outer sequence for both unconstrained and constrained settings in contrast to ergodic rates which are common in ALM as well as alternating direction method-of-multipliers literature. Compared to existing inexact ALM or quadratic penalty methods, our analysis does not rely on the worst-case bounds of the subproblem solved by the inner loop. Therefore, our algorithm can be viewed as a restarting technique applied to the ASGARD method in 58 but with rigorous theoretical guarantees or as an inexact ALM with explicit inner loop termination rules and adaptive parameters. Our algorithm only requires to initialize the parameters once, and automatically update them during the iteration process without tuning. We illustrate the superiority of our methods via several examples as compared to the state-of-the-art.
\end{abstract}

Keywords primal-dual first-order methods · restarting · augmented Lagrangian . homotopy $\cdot$ nonsmooth convex optimization $\cdot$ constrained convex programming

Mathematics Subject Classification (2000) 90C25 $\cdot 90 \mathrm{C} 06 \cdot 90-08$

Quoc Tran-Dinh

Department of Statistics and Operations Research, University of North Carolina at Chapel Hill (UNC-Chapel Hill), 333 Hanes Hall, CB\#3260, UNC Chapel Hill, NC 27599-3260.

E-mail: quoctd@email.unc.edu

Ahmet Alacaoglu • Volkan Cevher

Laboratory for Information and Inference Systems (LIONS), École Polytechnique Fédérale de Lausanne (EPFL), CH1015-Lausanne, Switzerland.

E-mail: \{ahmet.alacaoglu, volkan.cevher\}@epfl.ch

Olivier Fercoq

LTCI, Télécom ParisTech, Université Paris-Saclay, 75634-Paris, France.

E-mail: olivier.fercoq@etelecom-paristech.fr 


\section{Introduction}

We study the following nonsmooth composite convex minimization template:

$$
P^{\star}:=\min _{x \in \mathbb{R}^{p}}\{P(x):=f(x)+g(A x)\},
$$

where both $f: \mathbb{R}^{p} \rightarrow \mathbb{R} \cup\{+\infty\}$ and $g: \mathbb{R}^{n} \rightarrow \mathbb{R} \cup\{+\infty\}$ are proper, closed, and nonsmooth convex functions, and $A: \mathbb{R}^{p} \rightarrow \mathbb{R}^{n}$ is a linear operator.

Under only convexity and zero duality gap assumptions, the state-of-theart methods for solving (1) include primal-dual first-order methods (PDFOM) [2,13], and augmented Lagrangian-based algorithms [5,10]. While PDFOM directly tackles problem (1), the augmented Lagrangian-based framework (ALM) and its variants solve (1) via a constrained reformulation as follows:

$$
P^{\star}:=\min _{x \in \mathbb{R}^{p}, z \in \mathbb{R}^{n}}\{P(x, z):=f(x)+g(z) \text { s.t. } A x-z=0\},
$$

Alternating direction method of multipliers (ADMM) is another (and perhaps the most) successful method to solve (2). ADMM can be viewed as an approximation to ALM by alternating between $x$ and $z$ to break the computational bottleneck in the primal subproblem. Inexact and linearized variants enhance the scalability of ALM and ADMM for the same problem template [67,50,68].

While ADMM and PDFOM and their variants work really well in practice, their best-known convergence rate is $\mathcal{O}\left(\frac{1}{k}\right)$ under only convexity and zero duality gap assumptions, where $k$ is the iteration counter. Moreover, such a rate is achieved via an ergodic sense (i.e., using an averaging sequence or a weighted averaging sequence) $13,14,21,20,40,39,55]$.

In stark contrast, empirical evidence shows that averaging sequences in PDFOM and ALM exhibit the theoretical worst case rate $\mathcal{O}\left(\frac{1}{k}\right)$ in practice compared to the last iterate of the algorithm (see Subsection 4.1 for a concrete example), which is superior and often locally linear in many examples ${ }^{1}$ However, for these methods, last iterate generally has convergence guarantees but has no rate guarantees.

Recently, [58] proposed an accelerated, smoothed gap reduction (ASGARD) framework to solve nonsmooth convex optimization problems. ASGARD combines acceleration, smoothing, and homotopy techniques to handle both unconstrained and constrained nonsmooth problems, including (1).

One notable feature of ASGARD is a non-ergodic optimal $\mathcal{O}\left(\frac{1}{k}\right)$ rate on the objective residual, and feasibility violation in the constrained settings. Moreover, this method only requires one proximal operator of $f$, one matrix-vector multiplication, and one adjoint operator per iteration. When $f$ is separable, the algorithm can be naturally parallelized. However, as also noted in [58], ASGARD needs restarting to be competitive with state-of-the-art methods such as ADMM and PDFOM in practice. This is not surprising since empirical evidence [28,31, 48,56] has shown that restarting significantly improves the actual convergence rate in practice. While there exists theory to support the

1 There exist examples showing arbitrarily slow convergence rate of ADMM, see, e.g., [21]. 
restarting strategies in accelerated gradient-type methods, supporting theory of these strategies are not yet investigated in primal-dual methods.

In this paper, we introduce an analysis framework for restarting ASGARD and prove the same worst-case $\mathcal{O}\left(\frac{1}{k}\right)$ rate in a non-ergodic sense. To our knowledge, this is the first time restarting is studied for primal-dual methods with a rigorous convergence rate guarantee. While doing so, we identify that restarting ASGARD corresponds to an inexact ALM algorithm in the constrained case. In contrast to existing works on this front, our method has explicit inner-loop termination rules and does not need to set a horizon (i.e., the maximum number of inner iterations or a predefined inner loop accuracy) for the algorithm.

As a result, we present a method which has the guarantees on the last iterate compared to ALM/ADMM methods and extend the guarantees of ASGARD to the restarting case which significantly improves the practical performance. In addition, we allow general Bregman distances to be used for smoothing and proximal operators in contrast to the original ASGARD scheme. A more thorough discussion and comparison between our method and existing state-of-the-arts is deferred to Section 5 for the sake of presentation.

Composite vs. constrained settings: An interesting instance of (1) is the following constrained convex setting:

$$
f^{\star}:=\min _{x \in \mathbb{R}^{p}}\{f(x) \mid A x-b \in \mathcal{K}\},
$$

where $\mathcal{K}$ is a nonempty, closed, and convex set in $\mathbb{R}^{n}$, and $b \in \mathbb{R}^{n}$. If we define $g(u):=\delta_{b+\mathcal{K}}(u)$, the indicator of $b+\mathcal{K}$, then $(3)$ can be converted into (1).

In the general setting (1), under different choices of $f$ and $g,(1)$ covers a wide range of applications from different fields including compressive sensing, image/signal processing, machine learning, statistics, and optimal control. Classical and well-known examples such as LASSO, square-root LASSO, support vector machines, image denoising and deblurring, and matrix completion can be cast into (1), see, e.g., 10, 18, 51,66 for some concrete examples.

For the setting (3), we do not impose any restriction on $\mathcal{K}$. Hence, it covers a large class of constrained problems including equality and inequality constraints. When $\mathcal{K}$ is a given cone (e.g., $\mathbb{R}_{+}^{n}$, second-order cone, or symmetric positive semidefinite cone), problem (3) covers also problems with cone constraints such as linear programming, second-order cone, and semidefinite programming. Although the theory for (1) as well as for (3) are well developed, various numerical methods for solving these problems rely on different structure assumptions and do not have a unified analysis: $c f$., Section 5

Contributions: Our contributions can be summarized as follows.

(a) We propose a new self-adaptive, double-loop smoothing algorithm to solve nonsmooth convex optimization problems of the form (1). Our algorithm is based on Nesterov's smoothing technique via general Bregman distance functions. It self-adaptively selects the number of iterations in the inner loop to achieve a desired complexity bound without requiring the accuracy a priori as in variants of ALM. Compared to ASGARD [58], it incorporates 
restarts, updates the dual center, and can work with general Bregman distances instead of only Lipschitz gradient distances.

(b) We prove $\mathcal{O}\left(\frac{1}{k}\right)$-convergence rate on the last iterate of the outer sequence for both unconstrained and constrained settings in contrast to ergodic rates which are common in ALM/ADMM literature. This rate is known to be optimal [4, 47,65] under just convexity and strong duality assumptions. Compared to existing inexact ALM or quadratic penalty methods such as 42,69, our analysis does not rely on the worst-case bounds of the subproblem solved by the inner loop. Therefore, our algorithm can be viewed as a restarting technique applied to ASGARD but with rigorous theoretical guarantees or as an inexact ALM with explicit inner loop termination rules and adaptive parameters.

(c) As an upshot, we customize our algorithm to solve general constrained problems of the form (3). We prove the same $\mathcal{O}\left(\frac{1}{k}\right)$-convergence rate guarantee on both the objective residual $\left|f\left(x^{k}\right)-f\left(x^{\star}\right)\right|$ and the feasibility $\operatorname{dist}_{\mathcal{K}}\left(A x^{k}-b\right)$. This rate is given on the last iterate of the outer sequence.

Our algorithm is a primal-dual method, which can solve composite convex problem with linear operators as in Chambolle-Pock's method [13. It only requires one proximal operator of $f$ and $g^{*}$, one matrix-vector multiplication and one adjoint for each iteration. It is parallelizable when $f$ is separable, i.e., $f(x)=\sum_{i=1}^{N} f_{i}\left(x_{[i]}\right)$. Under this structure, our method has more advantages than ADMM and Chambolle-Pock's method. In the algorithm, we provide explicit rules to update all algorithmic parameters. We also note that these updates can be modified to trade-off between the primal or the dual progress.

Paper organization: The rest of this paper is organized as follows. Section[ 2 recalls some mathematical background and the ASGARD algorithm in 58. Section 3 presents our main result with algorithm and its convergence guarantee. We study both unconstrained and constrained cases. Section 3.3 shows an extension of our method to three composite objective functions with linearization on potentially smooth terms. We provide overall complexity bounds in Section 3.4 for linear programming and discuss their superiority compared to existing results. In Section 4, we provide seven numerical examples to test our algorithm against state-of-the arts. Section 5 compares our method and existing algorithms in the literature.

\section{Mathematical tools and ASGARD}

We review some key ingredients for the design of our primal-dual methods. We also recall the ASGARD algorithm in [58] and discuss its possible variants.

Notation: We denote the norm in primal space $\mathcal{X}$ as $\|\cdot\|_{\mathcal{X}}$ and the norm in dual space $\mathcal{Y}$ as $\|\cdot\|_{\mathcal{Y}}$. Their dual norms are denoted as $\|\cdot\|_{\mathcal{X}, *}$ and $\|\cdot\|_{\mathcal{Y}, *}$, respectively. Given a proper, closed, and convex function $f$, we use $\operatorname{dom}(f)$ to denote its domain and $\partial f(x)$ to denote its subdifferential at $x$. When the function is differentiable, we denote its gradient at $x$ as $\nabla f(x)$. For a given nonempty, closed, and convex set $\mathcal{K}$, we denote its indicator 
function as $\delta_{\mathcal{K}}(x)=0$, if $x \in \mathcal{K}, \delta_{\mathcal{K}}(x)=+\infty$, otherwise; and its support function as $s_{\mathcal{K}}(y)=\sup _{x \in \mathcal{K}}\langle x, y\rangle$. We define the normal cone of $\mathcal{K}$ as $\mathcal{N}_{\mathcal{K}}(x):=\left\{w \in \mathbb{R}^{n} \mid\langle w, y-x\rangle \geq 0, y \in \mathcal{K}\right\}$ if $x \in \mathcal{K} ; \mathcal{N}_{\mathcal{K}}(x):=\emptyset$, otherwise. We also define $\mathcal{K}^{o}:=\left\{w \in \mathbb{R}^{n} \mid\langle w, x\rangle \leq 1, x \in \mathcal{K}\right\}$ as the polar set of $\mathcal{K}$. If $\mathcal{K}$ is a convex cone, then $\mathcal{K}^{o}=-\mathcal{K}^{*}$, where $\mathcal{K}^{*}:=\left\{w \in \mathbb{R}^{n} \mid\langle w, x\rangle \geq 0, x \in \mathcal{K}\right\}$ the dual cone of $\mathcal{K}$. The Fenchel conjugate of a function $f$ is defined as $f^{*}(y):=\sup _{x}\{\langle x, y\rangle-f(x)\}$. We say that $f: \mathcal{X} \rightarrow \mathbb{R}$ has Lipschitz gradient if it satisfies $\|\nabla f(x)-\nabla f(y)\|_{\mathcal{X}, *} \leq L_{f}\|x-y\|_{\mathcal{X}}$, for any $x, y \in \mathcal{X}$. This is equivalent to $f(x) \leq f(y)+\langle\nabla f(y), x-y\rangle+\frac{L_{f}}{2}\|x-y\|_{\mathcal{X}}^{2}$, for all $x, y \in \mathcal{X}$. Given a positive real number $a,\lfloor a\rfloor$ denotes the largest integer that is less than or equal to $a$.

Given a proper, closed, and convex function $f: \mathbb{R}^{p} \rightarrow \mathbb{R} \cup\{+\infty\}, \operatorname{prox}_{f}(x):=$ $\operatorname{argmin}_{u}\left\{f(u)+(1 / 2)\|u-x\|_{\mathcal{X}}^{2}\right\}$ is called the proximal operator of $f$. We say that $f$ is "proximally tractable" if prox $_{f}$ can be computed efficiently, e.g., in a closed form, or by a polynomial algorithm. By Moreau's identity, we have $\operatorname{prox}_{\gamma f}(x)+\gamma \operatorname{prox}_{f^{*} / \gamma}\left(\gamma^{-1} x\right)=x$ for any $x \in \operatorname{dom}(f)$.

\subsection{Primal-dual formulation}

Dual problem and min-max formulation: Associated with the primal problem (1), we also consider the corresponding dual problem:

$$
D^{\star}:=\min _{y \in \mathbb{R}^{n}}\left\{D(y):=f^{*}\left(-A^{\top} y\right)+g^{*}(y)\right\}
$$

where $f^{*}$ and $g^{*}$ are the Fenchel conjugates of $f$ and $g$, respectively. Clearly, we can write the primal and dual pair (1)-(4) in the following min-max saddle point problem:

$$
\begin{aligned}
P^{\star} & =\min _{x \in \mathbb{R}^{p}} \max _{y \in \mathbb{R}^{n}}\left\{f(x)+\langle A x, y\rangle-g^{*}(y)\right\} \\
& =\max _{y \in \mathbb{R}^{n}} \min _{x \in \mathbb{R}^{p}}\left\{-g^{*}(y)-\left(\left\langle x,-A^{\top} y\right\rangle-f(x)\right)\right\}=-D^{\star}
\end{aligned}
$$

Under mild and standard assumptions, this min-max problem is solvable and achieves zero duality gap, i.e., $P^{\star}+D^{\star}=0$. In particular, the dual problem of (3) can be written as follows:

$$
D^{\star}:=\min _{y \in \mathbb{R}^{n}}\left\{D(y):=f^{*}\left(-A^{T} y\right)+\langle b, y\rangle+s_{\mathcal{K}}(y)\right\}
$$

where $s_{\mathcal{K}}(y)=\sup _{x \in \mathcal{K}}\langle y, x\rangle$ is the support function of $\mathcal{K}$. Compared to (4), we have $g^{*}(y)=\langle b, y\rangle+s_{\mathcal{K}}(y)=s_{b+\mathcal{K}}(y)$. Let $\mathcal{X}^{\star}$ and $\mathcal{Y}^{\star}$ be the solution sets of the primal problem (1) (or (3)) and dual problem (4) (or (6)), respectively.

Fundamental assumptions: Throughout this paper, we will develop methods for solving (1) and (3). Note that we will use different assumptions for these two cases, which are given below for (1) and (3), respectively. 
Assumption 2.1. The solution set $\mathcal{X}^{\star}$ of (1) is nonempty. Both $f$ and $g$ are proper, closed, and convex. Moreover, $\operatorname{dom}\left(g^{*}\right)$ is bounded, or equivalently, $g$ is Lipschitz continuous. Note that this implies the Slater condition $\mathbf{0} \in$ ri $(\operatorname{dom}(g)-A(\operatorname{dom}(f)))$, where $\operatorname{ri}(\mathcal{X})$ is the relative interior of $\mathcal{X}$.

Assumption 2.2. The solution set $\mathcal{X}^{\star}$ of $(3)$ is nonempty. The function $f$ is proper, closed, and convex. The constraint set $\mathcal{K}$ is nonempty, closed, and convex, and $\mathbf{0}^{n} \in \mathcal{K}$. The Slater condition $\operatorname{ri}(\operatorname{dom}(f)) \cap\left\{x \in \mathbb{R}^{p} \mid A x-b \in \operatorname{ri}(\mathcal{K})\right\} \neq$ $\emptyset$ holds, where ri $(\mathcal{X})$ is the relative interior of $\mathcal{X}$.

Except for the boundedness of $\operatorname{dom}\left(g^{*}\right)$, Assumptions 2.1 and 2.2 are very standard in convex optimization. It guarantees the strong duality of (1) (respectively, (3) and (4) to hold. The boundedness of $\operatorname{dom}\left(g^{*}\right)$ is guaranteed if and only if $g$ is Lipschitz continuous as we mentioned. We emphasize that we need boundedness of $\operatorname{dom}\left(g^{*}\right)$ only for (1) and we do not require it for (3)

We note that the assumption $\mathbf{0}^{n} \in \mathcal{K}$ is not restrictive, since if $\mathbf{0}^{n} \notin \mathcal{K}$, we can fix any point $\boldsymbol{e} \in \mathcal{K}$, and consider the set $\tilde{\mathcal{K}}=\mathcal{K}-\boldsymbol{e}$, then $\mathbf{0}^{n} \in \tilde{\mathcal{K}}$, and $A x-b \in \mathcal{K}$ becomes $A x-b+e \in \tilde{\mathcal{K}}$. Note that, in the sequel, we will refer to the setting of (1) with Assumption 2.1 as the bounded dual domain case, and to the setting of (3) with Assumptions 2.2 as the constrained case.

Optimality conditions: Associated with the primal and dual problems (1)(4), we have the following optimality conditions:

$$
0 \in \partial f\left(x^{\star}\right)+A^{\top} \partial g\left(A x^{\star}\right) \text { and } 0 \in-A \partial f^{*}\left(-A^{\top} y^{\star}\right)+\partial g^{*}\left(y^{\star}\right) .
$$

From (5), it is straightforward to see the relation $y^{\star} \in \partial g\left(A x^{\star}\right) \Leftrightarrow A x^{\star} \in$ $\partial g^{*}\left(y^{\star}\right)$ and $x^{\star} \in \partial f^{*}\left(-A^{\top} y^{\star}\right) \Leftrightarrow-A^{\top} y^{\star} \in \partial f\left(x^{\star}\right)$, we can write this optimality condition into the following KKT condition:

$$
0 \in \partial f\left(x^{\star}\right)+A^{\top} y^{\star} \quad \text { and } \quad 0 \in-A x^{\star}+\partial g^{*}\left(y^{\star}\right) .
$$

For the constrained problem (3) these conditions are written as

$$
0 \in \partial f\left(x^{\star}\right)+A^{\top} y^{\star}, \quad A x^{\star}-b \in \mathcal{K}, \quad \text { and } y^{\star} \in \mathcal{N}_{\mathcal{K}}\left(A x^{\star}-b\right),
$$

where $\mathcal{N}_{\mathcal{K}}(\cdot)$ is the normal cone of $\mathcal{K}$ defined above. If $\mathcal{K}$ is a closed, pointed, and convex cone, then $\mathcal{N}_{\mathcal{K}} \equiv-\mathcal{K}^{*}$ the dual cone of $\mathcal{K}$. In this case, $y^{\star} \in-\mathcal{K}^{*}$.

\subsection{Bregman Distances and Generalized Proximal Operators}

In the sequel, we will use Bregman distances for smoothing and computing proximal operators. Therefore, we give basic properties on Bregman distances.

Let $p_{\mathcal{Z}}$ be $\mu_{p}$-strongly convex, continuous, and differentiable on $\mathcal{Z}$ with the strong convexity $\mu_{p}=1$, where $\mathcal{Z}=\operatorname{dom}\left(p_{\mathcal{Z}}\right)$. We call $p_{\mathcal{Z}}$ a proximity function (or prox-function). We define the Bregman distance induced by $p_{\mathcal{Z}}$ as

$$
b_{\mathcal{Z}}(x, y):=p_{\mathcal{Z}}(x)-p_{\mathcal{Z}}(y)-\left\langle\nabla p_{\mathcal{Z}}(y), x-y\right\rangle, \quad \forall x, y \in \mathcal{Z}
$$

We assume that $b_{\mathcal{Z}}$ is 1 -strongly convex with respect to the norm $\|\cdot\|_{\mathcal{Z}}$. Then

$$
b_{\mathcal{Z}}(x, y) \geq \frac{1}{2}\|x-y\|_{\mathcal{Z}}^{2}, \quad \forall x, y \in \mathcal{Z} .
$$


A special case of prox-functions is $p_{\mathcal{Z}}(x)=\frac{1}{2}\|x\|_{2}^{2}$, which corresponds to the well-known Euclidean distance $b_{\mathcal{Z}}(x, y)=\frac{1}{2}\|x-y\|_{2}^{2}$. Another example is the entropy function $p_{\mathcal{Z}}(x):=\sum_{i} x_{i} \ln \left(x_{i}\right)$, which corresponds to the so-called KL divergence $b_{\mathcal{Z}}(x, y):=\sum_{i} x_{i} \ln \left(\frac{x_{i}}{y_{i}}\right)-x_{i}+y_{i}$. When a Bregman distance $b_{\mathcal{Z}}$ has Lipschitz continuous gradient, we denote its Lipschitz constant by $L_{b_{\mathcal{Z}}}$.

We also define the strong convexity of a function $f$ with respect to a proxfunction $p_{\mathcal{Z}}$ which induces the Bregman distance $b_{\mathcal{Z}}$ as follows:

$$
f(x) \geq f(y)+\langle\nabla f(y), x-y\rangle+b_{\mathcal{Z}}(x, y), \quad \forall x, y \in \mathcal{Z} .
$$

We refer to [16,24,33. for several concrete examples of Bregman divergences.

2.3 Nesterov's smoothing technique

We focus on Nesterov's smoothing technique with general Bregman distances 4,47. Since $g$ is nonsmooth, assuming that it admits a max-form as

$$
g(u)=\max _{y \in \mathcal{Y}}\left\{\langle u, y\rangle-g^{*}(y)\right\}, \quad \text { where } \mathcal{Y}=\operatorname{dom}\left(g^{*}\right),
$$

we smooth it by

$$
g_{\beta}(u ; \dot{y}):=\max _{y \in \mathcal{Y}}\left\{\langle u, y\rangle-g^{*}(y)-\beta b \mathcal{Y}(y, \dot{y})\right\}
$$

where $\dot{y} \in \mathbb{R}^{n}$ is a given center point, and $\beta>0$ is a smoothness parameter. The function $g_{\beta}(\cdot ; \dot{y})$ is convex and smooth, its gradient is given by

$$
\nabla g_{\beta}(u ; \dot{y})=y_{\beta}^{*}(u ; \dot{y})=\underset{y \in \mathcal{Y}}{\operatorname{argmin}}\left\{g^{*}(y)-\langle u, y\rangle+\beta b_{\mathcal{Y}}(y, \dot{y})\right\} .
$$

Clearly, $\nabla g_{\beta}(\cdot ; \dot{y})$ is Lipschitz continuous with the Lipschitz constant $L_{g_{\beta}}=\frac{1}{\beta}$. Moreover, we have

$$
g_{\beta}(u ; \dot{y}) \leq g(u) \leq g_{\beta}(u ; \dot{y})+\beta D_{\mathcal{Y}},
$$

where $D_{\mathcal{Y}}:=\sup \left\{b_{\mathcal{Y}}(y, \dot{y}) \mid y \in \operatorname{dom}\left(g^{*}\right)\right\}$ is the prox-diameter of $g^{*}$. Here, $D \mathcal{Y}$ is finite if and only if $g$ is Lipschitz continuous with the Lipschitz constant $L_{g}:=\sqrt{2 D_{\mathcal{Y}}}$, i.e., $|g(u)-g(v)| \leq \sqrt{2 D_{\mathcal{Y}}}\|u-v\|$ for all $u, v \in \operatorname{dom}(g)$ due to 7. Proposition 4.4.6].

If we choose $b_{\mathcal{Y}}(y, \dot{y})=\frac{1}{2}\|y-\dot{y}\|_{2}^{2}$, then we can write $y_{\beta}^{*}(u ; \dot{y})$ as:

$$
\nabla g_{\beta}(u ; \dot{y})=\arg \min _{y \in \mathbb{R}^{n}}\left\{g^{*}(y)-\langle u, y\rangle+\frac{\beta}{2}\|y-\dot{y}\|^{2}\right\}=\operatorname{prox}_{g^{*} / \beta}\left(\dot{y}+\frac{1}{\beta} u\right) \text {. }
$$

Smoothing techniques are widely used in the literature, including $[4,8,9,22$, 41. The idea of smoothing is to approximate the original problem (1) by a (partially) smoothed problem. For example, in our setting, we smooth $g$ and consider the following smoothed problem:

$$
P_{\beta}^{\star}:=\min _{x \in \mathbb{R}^{p}}\left\{P_{\beta}(x ; \dot{y}):=f(x)+g_{\beta}(A x ; \dot{y})\right\} .
$$


We define the following generalized proximal operator with Bregman distance $d_{\mathcal{X}}$ induced by a prox-function $q_{\mathcal{X}}$ :

$$
\mathcal{P}_{\theta f}^{d_{\mathcal{X}}}(u, y):=\underset{v \in \mathcal{X}}{\operatorname{argmin}}\left\{f(v)+\langle y, v-u\rangle+\frac{1}{\theta} d_{\mathcal{X}}(v, u)\right\} .
$$

Note that the setup described in this and previous subsections will allow us to use different Bregman distances for smoothing as in 10 and computing the proximal operator as in (15), depending on the geometry of the problem. Given that the Bregman distance $d_{\mathcal{X}}$ is defined in $\mathcal{X}$ and $b_{\mathcal{Y}}$ is defined in $\mathcal{Y}$, we define the following operator norm of $A$ :

$$
\|A\|:=\max _{x \in \mathbb{R}^{p}}\left\{\frac{\|A x\|_{\mathcal{Y}, *}}{\|x\|_{\mathcal{X}}}\right\} .
$$

Different from [4,9,22,41,47, our strategy allows one to update the smoothness parameter $\beta$ gradually at each iteration. Similar work can be found in 8 , [4], which are also essentially different from ours as discussed in Section 5.

2.4 ASGARD: A primal-dual gap reduction framework

In [58, the authors proposed two primal-dual algorithms to solve (1). The first algorithm, ASGARD (Accelerated Smoothed Gap Reduction), can be viewed as a variant of FISTA [3] applied to the smoothed problem of (3). The second one, ADSGARD (Accelerated Dual Smoothed Gap Reduction) is a Nesterov's accelerated variant [45] applied to the smoothed problem of the dual (4).

ASGARD: Let us recall the first algorithm, ASGARD, from [58] as in Algorithm 1 for our further reference.

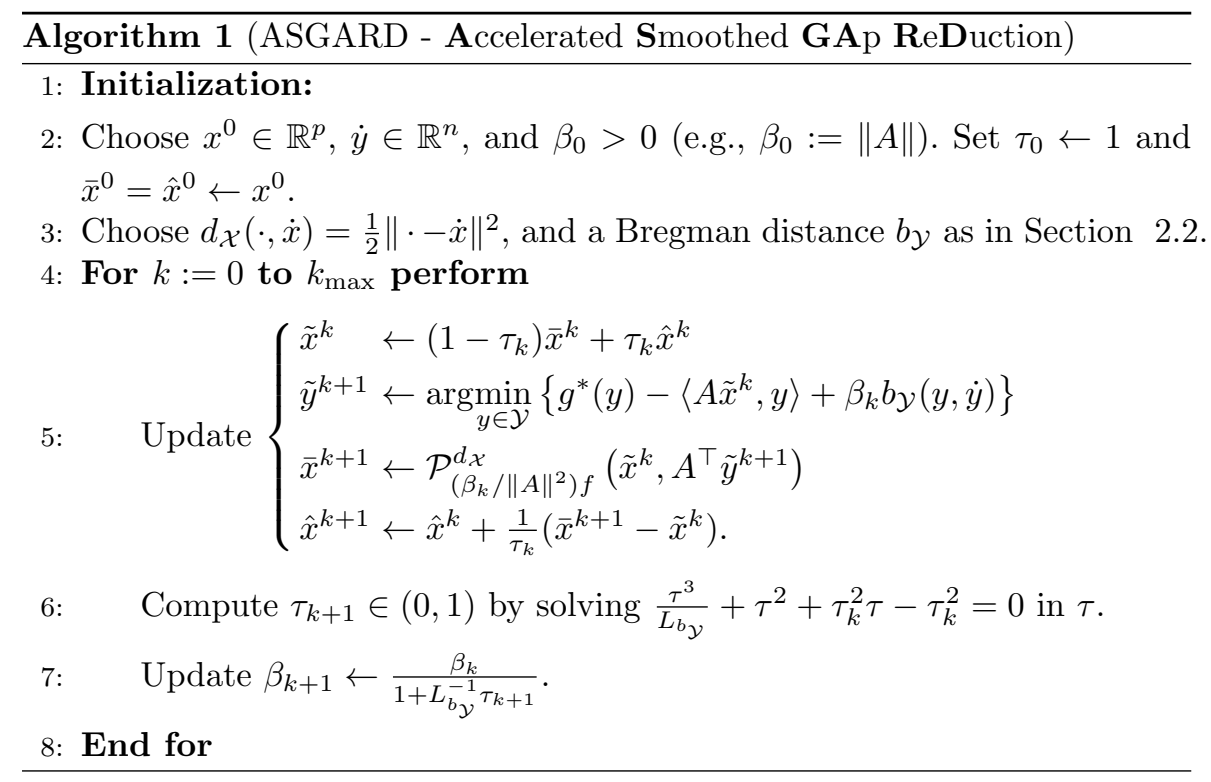


The main step of ASGARD, Algorithm 1, is Step 5, which requires one subproblem in $\tilde{y}^{k+1}$, one $\operatorname{prox}_{f}$ of $f$, one matrix-vector multiplication $A x$ and its adjoint $A^{\top} y$ at each iteration. If $b y(\cdot, \dot{y}):=\frac{1}{2}\|\cdot-\dot{y}\|_{2}^{2}$, then the computation of $\tilde{y}^{k+1}$ reduces to proximal operator $\operatorname{prox}_{g^{*}}$ of $g^{*}$. Therefore, the per-iteration complexity of Algorithm 1 is the same as in several primal-dual first-order algorithms 13,27. In contrast to existing works, the convergence guarantee is on the last primal iterate $\left\{\bar{x}^{k}\right\}$, instead of its weighted average $\left\{\tilde{x}^{k}\right\}$.

Variants: Although ASGARD relies on FISTA [3, one can replace Step 5 by any other accelerated proximal-gradient scheme such as Tseng's variant (APG) in [59]. One can also use two proximal operator schemes from 34,47] to substitute Step 5. To avoid the overload of this paper, we skip the analysis of Algorithm 1 and its variants, which can be found in [58].

Convergence: As proved in [58, if Algorithm 1 is applied to solve (1) with $b_{\mathcal{Y}}(\cdot ; \dot{y}):=\frac{1}{2}\|\cdot-\dot{y}\|_{\mathcal{Y}}^{2}$, then, under Assumption 2.1 , one has

$$
P\left(\bar{x}^{k}\right)-P^{\star} \leq \mathcal{O}\left(\frac{\|A\|\left\|x^{0}-x^{\star}\right\|_{\mathcal{X}} D_{\mathcal{Y}}}{k}\right) .
$$

If we apply Algorithm 1 to solve the constrained problem (3), then, under Assumption 2.2, we obtain the following guarantee

$$
\left\{\begin{array}{l}
\left|f\left(\bar{x}^{k}\right)-f^{\star}\right| \leq \mathcal{O}\left(\frac{\|A\|\left\|x^{0}-x^{\star}\right\|_{\mathcal{X}}\left\|y^{\star}\right\|_{\mathcal{Y}}}{k}\right) \\
\operatorname{dist}_{\mathcal{K}}\left(A \bar{x}^{k}-b\right) \leq \mathcal{O}\left(\frac{\|A\|\left\|x^{0}-x^{\star}\right\|_{\mathcal{X}}\left\|y^{\star}\right\|_{\mathcal{Y}}}{k}\right) .
\end{array}\right.
$$

Hence, the convergence rate of Algorithm 1 under Assumption 2.1 or Assumption 2.2 is $\mathcal{O}\left(\frac{1}{k}\right)$ and is in a non-ergodic sense.

\section{Main results: Self-Adaptive Double-Loop ASGARD}

In this section, we develop a self-adaptive double-loop accelerated smoothed primal-dual gap reduction algorithm to solve (1) and (3). We first present the complete algorithm. Next, we provide its convergence analysis. Then, we specify our algorithm to handle the constrained setting (3). Finally, we extend our method to handle (1) with the sum of three objective functions where the third function has Lipschitz gradient.

\subsection{The algorithm and its convergence guarantee}

Main idea: The proposed algorithm consists of two loops:

- The inner loop performs an accelerated proximal gradient scheme (APG) 59 to solve the smoothed problem (14) for a fixed $\beta$, which is a different strategy from [58, where $\beta$ is updated at each iteration. We note that in the constrained case, the smoothed problem $(14)$ is the augmented Lagrangian.

- The outer loop can be considered as a restarting step and simultaneously decreases the smoothness parameter $\beta$. 
The intuition behind our new strategy lies on the fact that when applied to 14 with a fixed $\beta$, APG gets $\mathcal{O}\left(\frac{1}{k^{2}}\right)$ rate, whereas ASGARD as presented in 58. controls the parameters in such a way that the algorithm gets $\mathcal{O}\left(\frac{1}{k}\right)$ rate throughout its execution. The idea is to take the advantage of the faster rate of APG for the inner loop while carefully adjusting the number of inner iterations and the smoothness parameter to get the same overall $\mathcal{O}\left(\frac{1}{k}\right)$ rate with better practical performance. Our analysis also gives insights on the heuristic restart strategy outlined in [58. For the sake of presentation and its flexibility for using Bregman distances in proximal operators, we choose Tseng's variant of APG [59. However, we can replace by another scheme such as FISTA 3. We adaptively determine the number of inner iterations at each outer iteration. Therefore, there is no need to tune this parameter. The outer loop gradually decreases the smoothness parameter $\beta$ such that the algorithm is still guaranteed to converge to the true solution of (1) or (3).

The algorithm: The complete algorithm is presented in Algorithm 2 .

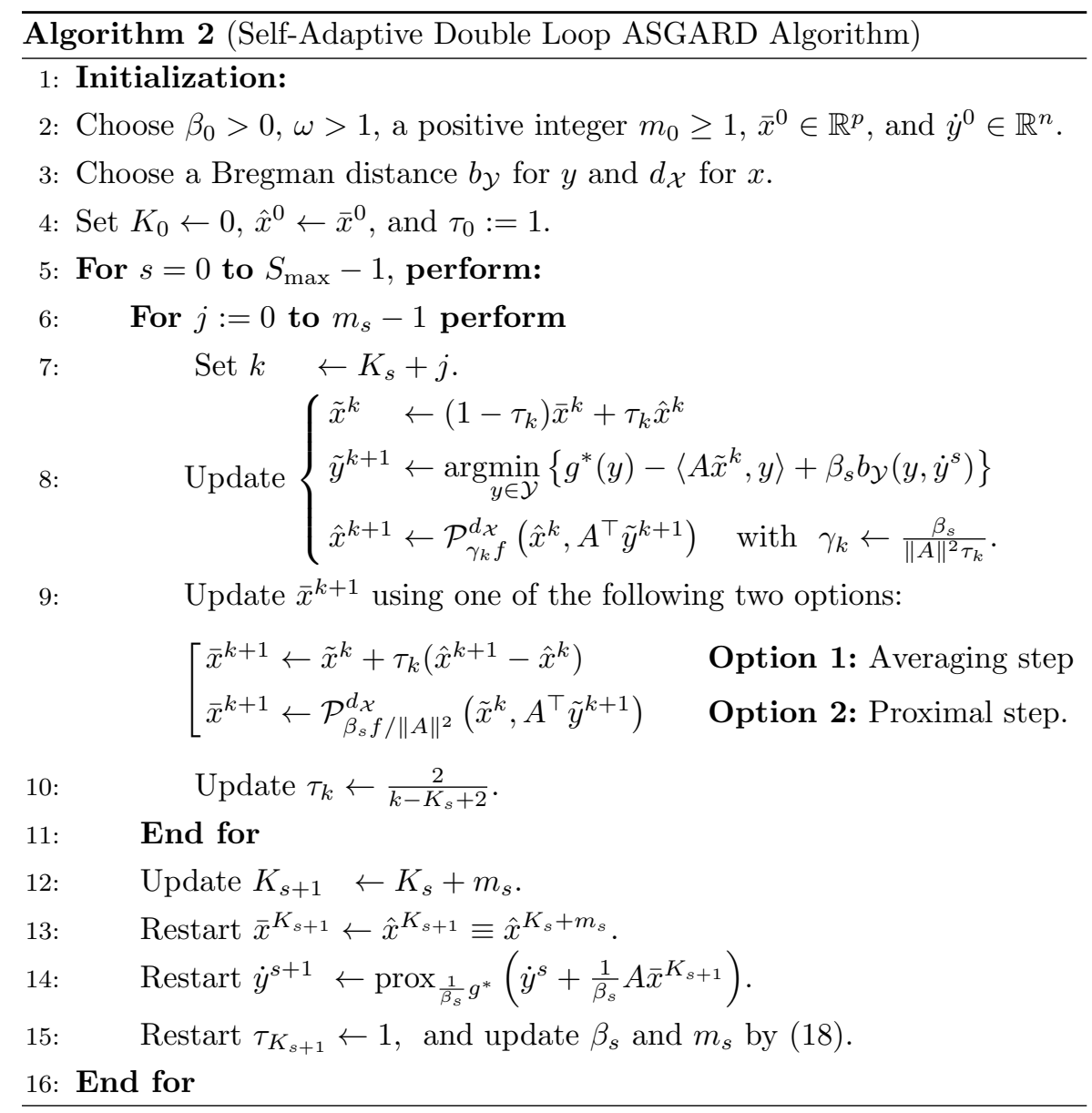


Algorithm 2 uses APG with Option 1 at Step 8 and has the same periteration complexity as Algorithm 1 except for the extra step, Step 14, where we update the dual center $\dot{y}^{s}$ at each outer loop iteration. In general, the number of outer iterations is small as it is the number of restarting steps. Hence, Step 14 does not significantly increase the overall computational cost of the entire algorithm. Note that $\bar{x}^{k+1}$ computed at Step 9 using Option 1 is a weighted averaging step. To avoid this averaging, we can choose Option 2, which requires an additional generalized proximal operator of $f$. Alternatively, we can replace Step 8 of Algorithm 2 by the following FISTA step:

$$
\left\{\begin{array}{l}
\tilde{y}^{k+1} \leftarrow \underset{y \in \mathcal{Y}}{\operatorname{argmin}}\left\{g^{*}(y)-\left\langle A \tilde{x}^{k}, y\right\rangle+\beta_{s} b_{\mathcal{Y}}\left(y, \dot{y}^{s}\right)\right\} \\
\bar{x}^{k+1} \leftarrow \mathcal{P}_{\gamma_{k} f}^{d \chi}\left(\tilde{x}^{k}, A^{\top} \tilde{y}^{k+1}\right) \quad \text { with } \gamma_{k} \leftarrow \frac{\beta_{s}}{\|A\|^{2}} \\
\tilde{x}^{k+1} \leftarrow \bar{x}^{k+1}+\frac{\left(1-\tau_{k}\right) \tau_{k+1}}{\tau_{k}}\left(\bar{x}^{k+1}-\bar{x}^{k}\right) .
\end{array}\right.
$$

However, we need to replace the general Bregman distance $d_{\mathcal{X}}$ by an Euclidean distance $d_{\mathcal{X}}(\cdot, \dot{x}):=\frac{1}{2}\|\cdot-\dot{x}\|^{2}$. The scheme (17) allows us to compute $\bar{x}^{k}$ through $\mathcal{P}_{\gamma_{k} f}^{d_{\mathcal{X}}}(\cdot)$ instead of a weighted averaging step as with Option 1.

Convergence guarantee: Now, we analyze convergence of Algorithm 2 for solving (1) under Assumption 2.1. Due to technical details, we separate the main theorem, Theorem 3.1 and its proof into different sections.

Theorem 3.1. Let $\left\{\bar{x}^{K_{s}}\right\}$ be the sequence generated by Algorithm 2 and $\omega>1$ be a given constant. Assume that (1) satisfies Assumption 2.1 and the parameters $\beta_{s}$ and $m_{s}$ are updated as

$$
\beta_{s+1} \leftarrow \frac{\beta_{s}}{\omega} \quad \text { and } \quad m_{s+1} \leftarrow\left\lfloor\omega\left(m_{s}+1\right)+1\right\rfloor-1 .
$$

where $m_{0} \geq 1$ and $\beta_{0}>0$. Let $\kappa_{0}:=m_{0}+\frac{\omega}{\omega-1}>0$. Then, we have

$$
P\left(\bar{x}^{K_{s+1}}\right)-P^{\star} \leq \frac{\omega \kappa_{0}}{\beta_{0}\left[(\omega-1) K_{s+1}+\kappa_{0}\right]}\left[R_{0}^{2}+\frac{\beta_{0}^{2} \omega D \mathcal{Y}}{(\omega-1) m_{0}}\right],
$$

where $R_{0}^{2}:=\frac{4\|A\|^{2}}{\left(m_{0}+1\right)^{2}} d_{\mathcal{X}}\left(x^{\star}, \bar{x}^{0}\right)+\beta_{0}^{2} b_{\mathcal{Y}}\left(y^{\star}, \dot{y}^{0}\right)$ and $D_{\mathcal{Y}}:=\sup \left\{b_{\mathcal{Y}}\left(y, \dot{y}^{s}\right) \mid y \in\right.$ $\left.\operatorname{dom}\left(g^{*}\right), \forall s \geq 0\right\}$.

Consequently, if $\operatorname{dom}\left(g^{*}\right)$ is bounded (equivalently, $g$ is Lipschitz continuous), then Algorithm 2 achieves $\mathcal{O}\left(\frac{1}{K_{s}}\right)$ convergence rate at the last iterate of the outer loop, i.e. $\left|P\left(\bar{x}^{K_{s+1}}\right)-P^{\star}\right| \leq \mathcal{O}\left(\frac{1}{K_{s+1}}\right)$.

We make a few remarks about Theorem 3.1 .

- The smoothness parameter $\beta$ is only updated at the outer loop but with a geometric rate, depending on the parameter $\omega$. We can select different $\omega$ to observe the performance in particular applications.

- The convergence rate depends on both the prox-distance between $\bar{x}^{0}$ to $x^{\star}$ and $\dot{y}^{0}$ to $y^{\star}$ as well as $D \mathcal{y}$, the prox-diameter of $\operatorname{dom}\left(g^{*}\right)$. 
- The convergence rate is given at the last iterate instead of the averaged sequence as often seen in other primal-dual methods [13, 49, 55, 67.

Remark 3.1. By using (48) in our analysis, we can show that $\left\{P\left(\bar{x}^{k}\right)\right\}$ converges to $P^{\star}$ at the rate of $\mathcal{O}\left(\frac{1}{k}\right)$ for any $k \geq 1$ instead of $k=K_{s}$ at the outer loop only.

- If we use the averaging step of APG, then $\bar{x}^{k}$ is computed via a weighted averaging step of the inner loop.

- However, if we use the proximal step in APG or the FISTA scheme (17), then $\bar{x}^{k}$ is computed through the generalized proximal operator $\mathcal{P}_{\beta_{s} f /\|A\|^{2}}^{d_{\mathcal{X}}}$. This rate is fully non-ergodic.

\subsection{Application to constrained convex optimization}

One important application of nonsmooth optimization is linearly constrained convex optimization. Most of the works on smoothing, including Nesterov's seminal work 47, need to know the diameter of the dual domain $D_{\mathcal{Y}}$ to set the smoothness parameter. For the case of linear equality constraints, $D_{\mathcal{Y}}$ is unbounded, therefore, these algorithms cannot be applied.

We will illustrate now how to apply our algorithm to constrained convex optimization problem (3), without any dependence on $D_{\mathcal{Y}}$. In this section, we require the Bregman distance used in smoothing for the dual variables to have Lipschitz gradient. Under this condition, we have

$$
b_{\mathcal{Y}}(y, \dot{y}) \leq \frac{L_{b_{\mathcal{Y}}}}{2}\|y-\dot{y}\|_{\mathcal{Y}}^{2} .
$$

Let us define $g(A x):=\delta_{\mathcal{K}}(A x-b)$ the indicator function of $\mathcal{K}$, where $\mathcal{K}$ satisfies Assumption 2.2. Then, we can write $g$ as

$$
g(A x):=\sup _{y \in \mathbb{R}^{n}}\left\{\langle A x-b, y\rangle-s_{\mathcal{K}}(y)\right\},
$$

where $s_{\mathcal{K}}(y):=\sup _{u \in \mathcal{K}}\langle y, u\rangle$ is the support function of $\mathcal{K}$. In this case, the smooth function $g_{\beta}(A x ; \dot{y})$ becomes

$$
g_{\beta}(A x ; \dot{y}):=\max _{y \in \mathbb{R}^{n}}\left\{\langle A x-b, y\rangle-s_{\mathcal{K}}(y)-\beta b_{\mathcal{Y}}(y, \dot{y})\right\} .
$$

Example 3.1. Suppose that $b_{\mathcal{Y}}(x, \dot{x})=\frac{1}{2}\|x-\dot{x}\|_{2}^{2}$. Then, the function $g_{\beta}(\cdot ; \dot{y})$ defined by 222 can be written as

$$
g_{\beta}(A x ; \dot{y})=\frac{1}{2 \beta} \operatorname{dist}_{\mathcal{K}}(A x-b+\beta \dot{y})^{2}-\frac{\beta}{2}\|\dot{y}\|^{2} .
$$

Moreover, the solution $y_{\beta}^{*}(A x ; \dot{y})$ of the maximization problem in 22 is given in a closed form as

$$
y_{\beta}^{*}(A x ; \dot{y})=\dot{y}+\frac{1}{\beta}\left(A x-b-\operatorname{proj}_{\mathcal{K}}(A x-b+\beta \dot{y})\right),
$$

where $\operatorname{proj}_{\mathcal{K}}(\cdot)$ denotes the projection onto $\mathcal{K}$. 
In particular, if $\mathcal{K}$ is a cone, then $y_{\beta}^{*}(A x ; \dot{y})=\operatorname{proj}_{-\mathcal{K}^{*}}\left(\dot{y}+\frac{1}{\beta}(A x-b)\right)$, where $\mathcal{K}^{*}$ is the dual cone of $\mathcal{K}$. The dual step for computing $\tilde{y}^{k}$ at the second line of Step 8 of Algorithm 2 becomes

$$
\begin{aligned}
\tilde{y}^{k+1} & \leftarrow \dot{y}^{s}+\frac{1}{\beta_{s}}\left(A \tilde{x}^{k}-b-\operatorname{proj}_{\mathcal{K}}\left(A \tilde{x}^{k}-b+\beta_{s} \dot{y}^{s}\right)\right) \\
& =\frac{1}{\beta_{s}} \operatorname{proj}_{-\mathcal{K}^{*}}\left(A \tilde{x}^{k}-b+\beta_{s} \dot{y}^{s}\right) .
\end{aligned}
$$

The following lemma provides a key estimate for the optimality condition of (3), whose proof is given in Appendix 7.2

Lemma 3.1. Let $S_{\beta}(\bar{x} ; \dot{y}):=f(\bar{x})+g_{\beta}(A \bar{x} ; \dot{y})-f\left(x^{\star}\right)$ and $\beta_{b}:=\beta L_{b y}$. Then:

$$
\begin{cases}f(\bar{x})-f^{\star} & \geq \beta_{b}\left\langle\dot{y}, y^{\star}\right\rangle-\left\|y^{\star}\right\| \operatorname{dist}_{\mathcal{K}}\left(A \bar{x}-b+\beta_{b} \dot{y}\right) \\ f(\bar{x})-f^{\star} & \leq S_{\beta}(\bar{x} ; \dot{y})-\frac{1}{2 \beta_{b}} \operatorname{dist}_{\mathcal{K}}\left(A \bar{x}-b+\beta_{b} \dot{y}\right)+\frac{\beta_{b}}{2}\|\dot{y}\|^{2} \\ \operatorname{dist}_{\mathcal{K}}\left(A \bar{x}-b+\beta_{b} \dot{y}\right) \leq \beta_{b}\left[\left\|y^{\star}\right\|+\left(\left\|\dot{y}-y^{\star}\right\|^{2}+\frac{2}{\beta_{b}} S_{\beta}(\bar{x} ; \dot{y})\right)^{1 / 2}\right] .\end{cases}
$$

Here, the term $\left\|\dot{y}-y^{\star}\right\|^{2}+\frac{2}{\beta_{b}} S_{\beta}(\bar{x} ; \dot{y}) \geq\left\|y_{\beta}^{*}(A \bar{x} ; \dot{y})-\dot{y}\right\|^{2} \geq 0$. In addition, we have the following bound for any $\beta$ :

$$
\begin{aligned}
\operatorname{dist}_{\mathcal{K}}(A \bar{x}-b)-\beta\left(\left\|\dot{y}-y^{\star}\right\|\right. & \left.+\left\|y^{\star}\right\|\right) \leq \operatorname{dist}_{\mathcal{K}}(A \bar{x}-b-\beta \dot{y}) \\
& \leq \operatorname{dist}_{\mathcal{K}}(A \bar{x}-b)+\beta\left(\left\|\dot{y}-y^{\star}\right\|+\left\|y^{\star}\right\|\right) .
\end{aligned}
$$

Now, we apply Algorithm 1 to solve the constrained convex problem (3). Then, the following steps are changed:

- The APG scheme at Steps 8 and 9 is replaced by the FISTA scheme 17).

- The dual step for computing $\tilde{y}^{k+1}$ of Algorithm 2 becomes

$$
\tilde{y}^{k+1} \leftarrow \arg \max _{y \in \mathbb{R}^{n}}\left\{\left\langle A \tilde{x}^{k}-b, y\right\rangle-s_{\mathcal{K}}(y)-\beta_{s} b y\left(y, \dot{y}^{s}\right)\right\} .
$$

- The update rule of $\tau_{k}$ and $\beta_{k}$ is changed as 29).

Combining the result of Theorem 3.1 and Lemma 3.1. we obtain the following convergence result of this new variant of Algorithm 2 .

Theorem 3.2. Assume that Assumption 2.2 holds. Let $\left\{\bar{x}^{K_{s}}\right\}$ be the sequence generated by Algorithm 1 for solving (3) using (28) for $\tilde{y}^{k+1}$. Let the parameters $\beta_{s}$ and $m_{s}$ be updated as

$$
\left\{\begin{array}{l}
m_{s+1} \leftarrow\left\lfloor\omega\left(m_{s}+1\right)+1\right\rfloor-1 \quad \text { with } \quad m_{0}>\frac{1}{\omega-1} \\
\beta_{s+1} \leftarrow \frac{\beta_{s}\left(m_{s+1}+1\right)}{\omega \sqrt{m_{s+1}\left(m_{s+1}+3\right)}} .
\end{array}\right.
$$

Then, we have

$$
\left\{\begin{array}{l}
f\left(\bar{x}^{K_{s+1}}\right)-f^{\star} \quad \geq-\left\|y^{\star}\right\| \operatorname{dist}_{\mathcal{K}}\left(A \bar{x}^{K_{s+1}}-b\right)-\frac{2 \sqrt{2} \omega \beta_{0} L_{b y} \kappa_{0}\left\|y^{\star}\right\| R_{0}}{\rho_{0}\left[(\omega-1) K_{s+1}+\kappa_{0}\right]} \\
f\left(\bar{x}^{K_{s+1}}\right)-f^{\star} \leq \frac{\omega \kappa_{0} R_{0}^{2}}{\rho_{0}\left[(\omega-1) K_{s+1}+\kappa_{0}\right]}+\frac{\omega \beta_{0} L_{b_{\mathcal{Y}}} \kappa_{0}}{2\left[(\omega-1) K_{s+1}+\kappa_{0}\right]}\left(\left\|y^{\star}\right\|^{2}+\frac{2 R_{0}^{2}}{\rho^{2}}\right) \\
\operatorname{dist}_{\mathcal{K}}\left(A \bar{x}^{K_{s+1}}-b\right) \leq \frac{\omega \beta_{0} L_{b_{y} \kappa_{0}}}{\left[(\omega-1) K_{s+1}+\kappa_{0}\right]}\left[2\left\|y^{\star}\right\|+\left(2 \sqrt{2}+\sqrt{\frac{2}{L_{b \mathcal{Y}}}}\right) \frac{R_{0}}{\rho_{0}}\right]
\end{array}\right.
$$


where $y^{\star}$ is any dual solution of (4), and

$$
\left\{\begin{array}{l}
\rho_{0}:=\beta_{0}\left(1-\frac{1}{(\omega-1) m_{0}}\right) \\
\kappa_{0}:=m_{0}+\frac{\omega}{\omega-1} \\
R_{0}:=\left[\frac{4\|A\|^{2}}{\left(m_{0}+1\right)^{2}} d \mathcal{X}\left(x^{\star}, \bar{x}^{0}\right)+\frac{\beta_{0}^{2} m_{0}\left(m_{0}+3\right)}{\left(m_{0}+1\right)^{2}} b_{\mathcal{Y}}\left(y^{\star}, \dot{y}^{0}\right)\right]^{1 / 2} .
\end{array}\right.
$$

Consequently, Algorithm 2] achieves an $\mathcal{O}\left(\frac{1}{K_{s}}\right)$ convergence rate in a nonergodic sense, i.e., $\left|f\left(\bar{x}^{K_{s}}\right)-f^{\star}\right| \leq \mathcal{O}\left(\frac{1}{K_{s}}\right)$ and $\operatorname{dist}_{\mathcal{K}}\left(A \bar{x}^{K_{s}}-b\right) \leq \mathcal{O}\left(\frac{1}{K_{s}}\right)$.

\subsection{Extension to Composite Case with Three Objective Terms}

It is straightforward to apply Algorithm 2 in the presence of a smooth term in the objective. The problem template we focus on in this section is

$$
F^{\star}:=\min _{x \in \mathbb{R}^{p}}\{F(x):=f(x)+g(A x)+h(x)\},
$$

where $f$ and $g$ are as described in Assumption 1 and $h$ is a differentiable function with $L_{h}$-Lipschitz gradient. In this case, only Step 8 in Algoritm 2 needs to be modified as follows (see also in [60]):

$$
\hat{x}^{k+1} \leftarrow \mathcal{P}_{\gamma_{k} f}^{d \mathcal{X}}\left(\hat{x}^{k}, \nabla h\left(\tilde{x}^{k}\right)+A^{\top} \tilde{y}^{k+1}\right) \quad \text { with } \gamma_{k} \leftarrow \frac{\beta_{s}}{\tau_{k}\left(\|A\|^{2}+\beta_{s} L_{h}\right)} .
$$

Note that, this modification only changes the analysis of the inner loop as in 60] which does not affect our analysis of the outer loop. In addition, using $L_{h}$ in the stepsize is not restrictive. When the Lipschitz constant is not known, line search strategies can be employed, see 60 for more details. The convergence of this variant is still guaranteed by Theorem 3.1 but the quantity $R_{0}^{2}$ will depend on $L_{h}$. We omit the details of this result here for succinctness.

\subsection{Better complexity bounds for Linear Programming}

As an application of our theory, we analyze the overall complexity of our algorithm for linear programming:

$$
\min _{x \in \mathbb{R}^{p}}\left\{c^{\top} x \quad \text { s.t. } \quad A x=b, x \geq 0\right\},
$$

where we define $f(x):=c^{\top} x+\delta_{\{x \geq 0\}}(x)$.

First-order methods for linear programming have been widely studied in the literature. However, it is generally not preferred since nonsmooth optimization methods have $\mathcal{O}\left(\frac{1}{\varepsilon^{2}}\right)$ complexity [54,63], where $\varepsilon$ is a desired accuracy. 54] proposed applying Nesterov smoothing to linear programs. Unfortunately, since Nesterov smoothing does not apply to indicator function of the linear constraints, their per-iteration complexity requires projection to the domain defined by the linear constraint which is computationally expensive. We propose applying smoothing to the linear constraint since our theory supports it to come up with an algorithm with much cheaper iterations. 
Given $f(x):=c^{\top} x+\delta_{\{x \geq 0\}}(x)$, we define $\tilde{x}^{*}$ to be an $\varepsilon$-solution to 32 if

$$
\left|f\left(\tilde{x}^{*}\right)-f\left(x^{\star}\right)\right| \leq \varepsilon \text { and }\left\|A \tilde{x}^{*}-b\right\| \leq \varepsilon .
$$

Using the bounds from Theorem 3.2 , we can derive the iteration complexity of our method for linear programming. Let us use $b \mathcal{Y}\left(y_{1}, y_{2}\right):=\frac{1}{2}\left\|y_{1}-y_{2}\right\|_{2}^{2}$ and $d_{\mathcal{X}}\left(x_{1}, x_{2}\right)=\frac{1}{2}\left\|x_{1}-x_{2}\right\|_{2}^{2}$ and let $\bar{x}^{0}$ and $\dot{y}^{0}$ to be all zero vectors. Then, we can achieve an $\varepsilon$-solution $x^{K}$ of 32 for any $K \geq C K_{\varepsilon}$, where

$$
K_{\varepsilon}=\frac{\max \left(\left\|y^{\star}\right\|^{2}, R_{0}^{2}\right)}{\varepsilon}=\frac{\max \left(\left\|y^{\star}\right\|^{2},\|A\|^{2}\left\|x^{\star}\right\|^{2}\right)}{\varepsilon},
$$

and $C$ hides dimension independent quantities. The per-iteration complexity of our method is dominated by applying $A$ matrix which has $\operatorname{nnz}(A)$ complexity. Combining this with the iteration complexity, we can estimate the overall complexity of our method for linear programming as

$$
\mathcal{O}\left(\max \left(\|A\|^{2}\left\|x^{\star}\right\|^{2},\left\|y^{\star}\right\|\right) \operatorname{nnz}(A)\left(\frac{1}{\varepsilon}\right)\right) .
$$

In 54, the author focuses on a specific case of linear programming where the primal domain diameter can be bounded. Here, we focus on general linear programs, therefore, it is fairer to compare our complexity with 63. Compared with 63 , from the overall complexity, we remove the terms $a_{m}:=\max _{i}\left\|A_{i}\right\|$ and $\theta_{S^{*}}^{2}$ where $\theta_{S^{*}}^{2}$ is the Hoffman bound and $S^{*}$ is the solution set. One thing to note here is that 63 . has a better dependence in terms of $\epsilon$ since they have linear convergence. Our method, has a sublinear rate for accuracy, but a better dimension dependence which is the main bottleneck in large scale linear programming.

\section{Numerical experiments}

We will test standard ASGARD 58,60, ASGARD with restart 58,60 and standard Chambolle-Pock's algorithm 13 on the following problems. Note that when there is a smooth term in the objective, we use the version of Chambolle-Pock which linearizes the smooth term, which is also known in the literature as Vu-Condat's algorithm 61, 19. We omit HOPS 69] from the comparisons because it does not apply to Basis pursuit and Markowitz's portfolio optimization problems due to the unboundedness of the dual domain. For the $\ell_{1}$-SVM example, we observed it to be extremely slow and difficult to tune for different datasets. In all the experiments, we have used the standard $b_{\mathcal{Y}}\left(y_{1}, y_{2}\right)=\frac{1}{2}\left\|y_{1}-y_{2}\right\|_{2}^{2}$ and $d_{\mathcal{X}}\left(x_{1}, x_{2}\right)=\frac{1}{2}\left\|x_{1}-x_{2}\right\|_{2}^{2}$ for smoothing and computing the proximal operators for fair comparison with other methods which do not allow Bregman distances. In the sequel, we refer to our algorithm as ASGARD-DL. In some cases, we also compare with ADMM and its variants.

The parameters are set as follows. For Chambolle-Pock's method, we set its step-size $\sigma=\tau=\frac{1}{\|A\|}$, where $A$ is the linear operator in (1). For ASGARDDL, we choose $\omega:=1.2$ and $m_{s}:=6$ which give us comparable performance. For restarting ASGARD, we set the restarting frequency to be $s=10$ in all experiments. 


\subsection{Convergence guarantees: Ergodic vs. Non-ergodic}

ALM, ADMM and Chambolle-Pock methods have the convergence rate guarantees in an ergodic sense. That is, they have the rate guarantees only on the averaged iterate sequence. In contrast, our guarantees are for the last iterate of the algorithm. To illustrate the importance between these two, we consider two synthetic problems in this section. The first one is a square root LASSO problem widely studied in the literature, which is given by:

$$
F^{\star}:=\min _{x \in \mathbb{R}^{p}}\left\{F(x):=\|A x-b\|_{2}+\lambda\|x\|_{1}\right\}
$$

where $A \in \mathbb{R}^{n \times p}$ is generated using a Gaussian distribution and is normalized such that column norms are equal to 1 . Given a groundtruth vector $x^{\natural}$, we generate the observations as $b=A x^{\natural}+\sigma \mathbf{n}$, where $\mathbf{n}$ is a noise vector generated by a standard Gaussian distribution and $\sigma=0.01$. We set $\lambda=0.03$ which is tuned to get a good recovery of $x^{\natural}$.

In this experiment, we test the ergodic and non-ergodic variants of Linearized ADMM (in the sense that the augmented term in the Lagrangian is linearized) [29], and Chambolle-Pock's algorithm [13]. These methods have convergence guarantees for their last iterates, however, their rate guarantees only apply to the averaged sequence. Moreover, they are very successful to solve this type of problems as can be seen from the litterature. The behavior of the algorithms is given in Figure 1.
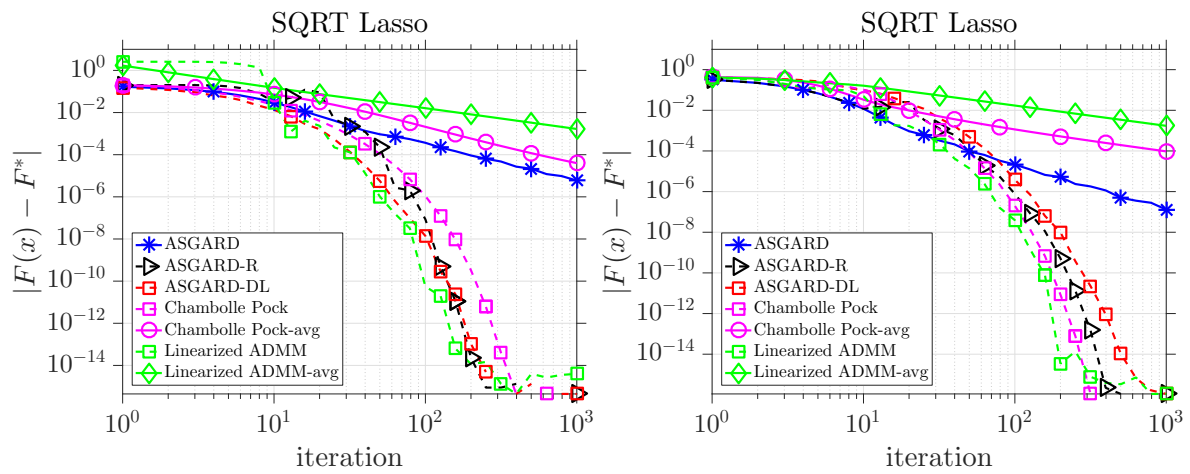

Fig. 1 Performance of 5 algorithms for solving square root LASSO problem. Left: $\sigma=$ $0.1, \lambda=0.04$, Right: $\sigma=0.01, \lambda=0.03$

As can be seen in Figure 1, last iterates of Linearized ADMM and Chambolle Pock's algorithms seem to have the best performance. However, the averaged iterates for which the methods have the rate guarantees shows the slowest convergence behavior. Our method has the same rate as restarted ASGARD which does not have any convergence guarantees.

To illustrate the behavior of the last iterates of Linearized ADMM and Chambolle Pock's algorithm, we consider a degenerate linear program which 
is also studied in 58 :

$$
\min _{x \in \mathbb{R}^{p}}\left\{h(x):=2 x_{p} \mid \sum_{k=1}^{p-1} x_{k}=1, \quad x_{p}-\sum_{k=1}^{p-1} x_{k}=0 \quad(2 \leq j \leq n), \quad x_{p} \geq 0\right\} .
$$

The second inequality is repeated $n-1$ times which causes the problem to be degenerate. We define the linear constraint as

$$
A x:=\left[\sum_{k=1}^{p-1} x_{k}, \quad x_{p}-\sum_{k=1}^{p-1} x_{k}, \cdots, \quad x_{p}-\sum_{k=1}^{p-1} x_{k}\right]^{\top} .
$$

We have $b:=(1,0, \cdots, 0)^{\top} \in \mathbb{R}^{n}$. We map the problem to our template in (31) as $f(x):=\delta_{\left\{x_{p} \geq 0\right\}}\left(x_{p}\right), g(x):=\delta_{\{b\}}(A x)$, and $h(x):=2 x_{p}$. For this problem, we pick $p=10$ and $n=200$.

In addition to Linearized ADMM and Chambolle-Pock's algorithm, we also include linearized ALM [29] to solve this example. The result of this test is given in Figure 2, where $F(x)=h(x)$.

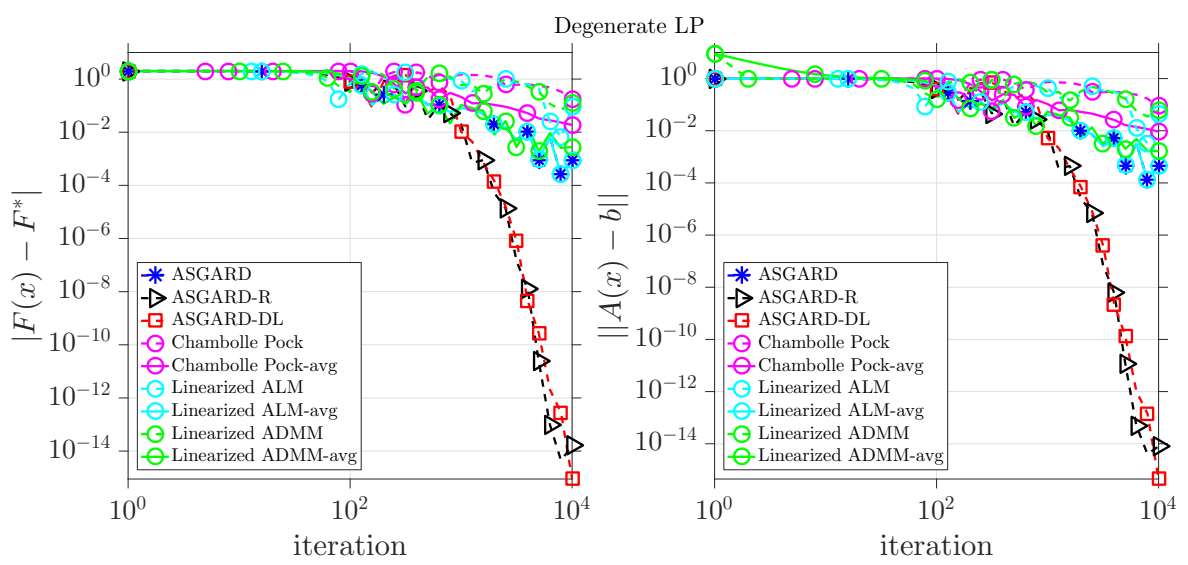

Fig. 2 Performance of 6 algorithms for solving the degenerate linear program.

As can be seen from Figure 2, Linearized ADMM, Linearized ALM and Chambolle-Pock's algorithm can get extremely slow where our algorithm and ASGARD with restart makes progress and converges to optimal value with a very high accuracy, and beyond the theoretical rate guarantee.

\subsection{Basis Pursuit for recovering Bag-of-Words of text documents}

We first consider a basis pursuit problem which is used in signal/image processing, statistics, and machine learning [17,23, 12]:

$$
\min _{x \in \mathbb{R}^{p}}\left\{F(x):=\|x\|_{1} \mid A x=b\right\},
$$

where $A \in \mathbb{R}^{n \times p}$ and $b \in \mathbb{R}^{n}$. This problem clearly fits into our template (1) by mapping $f(\cdot)=\|\cdot\|_{1}$ and $g(\cdot)=\delta_{\{b\}}(\cdot)$. It is also a special case of (3) with $\mathcal{K}=\{\mathbf{0}\}$. Proximal operators of both terms are given in a closed form. 
We apply this model to text processing. In [1, the authors proposed using basis pursuit formulation to obtain bag-of-words representation from the unigram embedding representation of a text. The setting can be briefly described as the following: For any word $w$, there exists a word vector $v_{w} \in \mathbb{R}^{n}$. For a given text document $\left\{w_{1}, \cdots, w_{T}\right\}$, one defines the unigram embedding as $\sum_{i=1}^{T} v_{w_{i}}$. It is easy to see that unigram embeddings can be written as a linear system $A x$ where $A \in \mathbb{R}^{n \times p}$ contains $v_{w_{i}}$ in the $i^{t h}$ column and $x \in \mathbb{R}^{p}$ is the bag-of-words vector which counts the number of occurances of words in a text. This application is considered in text processing applications to obtain the original text document given the unigram embeddings 64 .

For this experiment, we have used the movie review dataset of [38]. We have selected 4 different documents and computed the unigram embeddings using pre-trained word embeddings from GloVe [52] with $n=50$ as the dimension of the word vectors and restricted the vocabulary size to $p=10,000$ for getting faster results with all algorithms.

We have applied 4 methods to solve (35) for 4 different documents. Here, the parameter $\beta_{0}$ in ASGARD, ASGARD-restart, and ASGARD-DL is set to $\beta_{0}:=10\|A\|$. Note that this choice is not optimal, but give us reasonable results in all test. The results are compiled in Figure 3.

As we can observe from Figure 3 , our new algorithm works quite well and is comparable with state-of-the-art methods for low accuracy. It outperforms them if we run the algorithms long enough to get more accurate solutions than $\varepsilon=10^{-5}$ both in objective residual and feasibility. Note that (35) is fully nonsmooth, and $A$ is non-orthogonal. If we apply ADMM to solve (35), then it requires to solve a general convex subproblem, or a linear system, which has higher per-iteration complexity than four methods we used in this example.

\subsection{The $\ell_{1}$-Regularized Least Absolute Deviation Problem (LAD)}

Our second example is the $\ell_{1}$-regularized least absolute deviation regression problem, also known as LAD-Lasso in the literature. It is known that when the noise has a heavy tailed distribution such as Laplace distribution, LAD-Lasso is more robust to the outliers 62 . The optimization model of this problem is

$$
\min _{x \in \mathbb{R}^{p}}\left\{F(x):=\|A x-b\|_{1}+\lambda\|x\|_{1}\right\},
$$

where $A \in \mathbb{R}^{n \times p}$ is generated according to a normal distribution and the noise $\mathbf{n} \in \mathbb{R}^{n}$ is generated by Laplace $(0,1)$ distribution. We generate an observed vector $b:=A x^{\natural}+\sigma \mathbf{n}$, where $\sigma:=0.1$ and $x^{\natural}$ is a $s$-sparse vector of groundtruth. We choose $\lambda:=1 / n$ for the regularization parameter, which gives us a good recovery of $x^{\natural}$.

This problem fits to our template by setting $f(\cdot):=\lambda\|\cdot\|_{1}$ and $g(\cdot)=$ $\|\cdot-b\|_{1}$. We set $\beta_{0}$ in ASGARD, ASGARD-restart, and ASGARD-DL as $\beta_{0}:=100\|A\|$. We generated three problem instances of the size $n:=340 \mathrm{r}$, $p:=1000 r, s:=100 r$, where $s$ is the sparsity level, and $r=1,2,3$ for the first, second and third instances, respectively. We present the results of this example in Figure 4. 

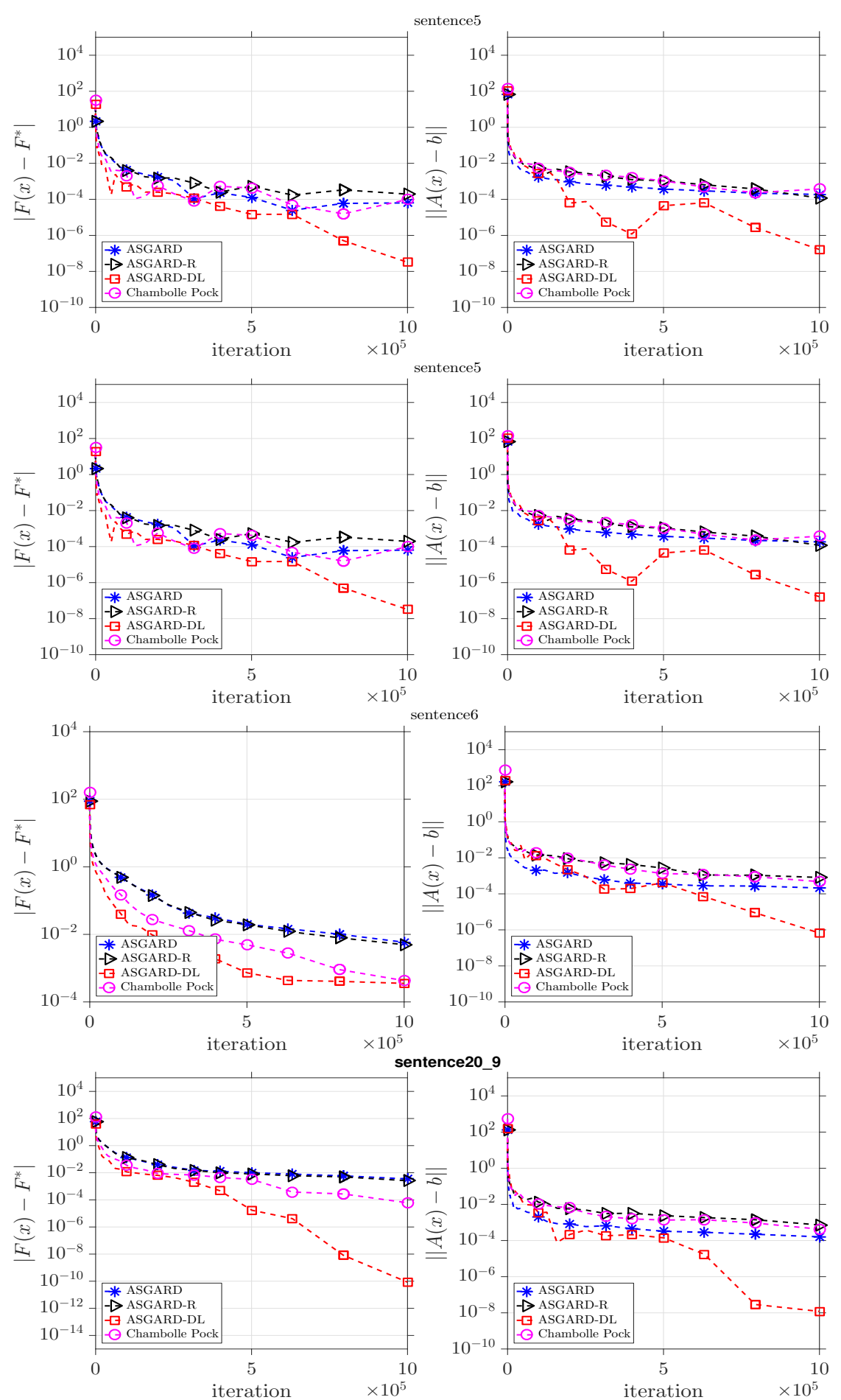

Fig. 3 Performance of 4 algorithms for solving basis pursuit for 4 text documents. 

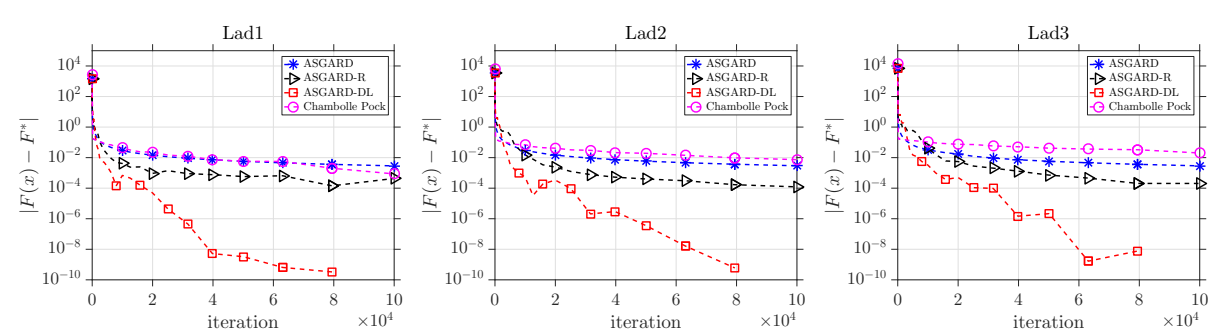

Fig. 4 Performance of 4 algorithms for LAD-Lasso problem in 3 different realization of varying problem size.

As we can see from Figure 4 that, with the same per-iteration complexity, our method significantly outperforms the other algorithms after accuracy $10^{-4}$. It beats other algorithms after a couple of hundred iterations and continues to decrease the objective values. Although this problem is fully nonsmooth, heuristic restart such as in ASGARD still improves the performance of the non-restart one, but does not significant outperform.

\subsection{Support Vector Machines}

Our next example is the following primal support vector machines (SVM) problem in binary classification. Instead of classical models, we consider the following $\ell_{1}$-regularized nonsmooth hinge loss as proposed in [70]:

$$
\min _{x \in \mathbb{R}^{p}}\left\{F(x):=\frac{1}{n} \sum_{i=1}^{n} \max \left\{0,1-b_{i}\left\langle a_{i}, x\right\rangle\right\}+\lambda\|x\|_{1}\right\},
$$

where $a_{i} \in \mathbb{R}^{p}$ are the feature vectors and $b_{i} \in\{-1,+1\}$ are the labels for $i=1, \cdots, n$. We can cast (37) into our template by setting $f(\cdot):=\lambda\|\cdot\|_{1}$ and

$$
g(A x)=\frac{1}{n} \sum_{i=1}^{n} \max \left\{0,1-b_{i}\left\langle a_{i}, x\right\rangle\right\}=\max _{y \in[0,1]^{n}}\left\langle y, A x+\frac{1}{n} \mathbb{1}\right\rangle,
$$

where $A:=-\frac{1}{n}\left[b_{1} a_{1}, b_{2} a_{2}, \cdots, b_{n} a_{n}\right]^{\top}$ and $\mathbb{1}$ is a vector of all ones. Clearly, the proximal operator of $g$ is simply a projection onto $[0,1]^{n}$.

We use 10 different datasets from libsvm [15] to test four different algorithms. The initial value $\beta_{0}$ in ASGARD, ASGARD-restart, and ASGARD-DL is set to $\beta_{0}:=0.1\|A\|$. But for covtype dataset, we used $\beta_{0}:=0.01\|A\|$. The details about the datasets are given in Table 1 . We test 4 algorithms on these ten datasets. The results of the first 8 problems are given in Figure 5 , and the results of the two last problems are in Figure 6 .

We again observe that Algorithm 2 significantly outperform the other methods. Since these algorithms have the same per-iteration complexity, it is sufficient to compare them in terms of iteration numbers. Although all the algorithms have $\mathcal{O}\left(\frac{1}{k}\right)$-worst-case convergence rate, due to its double-loop, Algorithm 2 performs much better than the others, especially for high accurate solutions. This is not surprise. The double-loop allows Algorithm 1 to use large stepsize by frequently restarting $\tau_{k}$ and $\beta_{k}$, while ASGARD gradually 
Table 1 Datasets used for classification.

\begin{tabular}{l|r|r}
\hline Data set & Training size & Number of features \\
\hline w1a & 2,477 & 300 \\
w2a & 3,470 & 300 \\
w3a & 4,912 & 300 \\
w4a & 7,366 & 300 \\
w5a & 9,888 & 300 \\
w6a & 17,188 & 300 \\
w7a & 24,692 & 300 \\
w8a & 49,749 & 300 \\
rcv1 & 20,242 & 47,236 \\
covtype & 581,012 & 54 \\
\hline
\end{tabular}

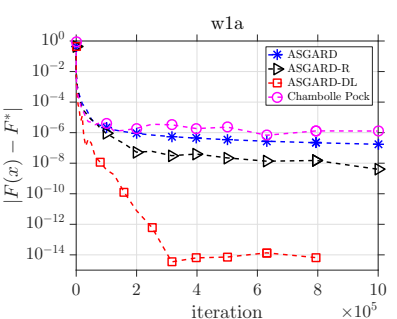

w4a

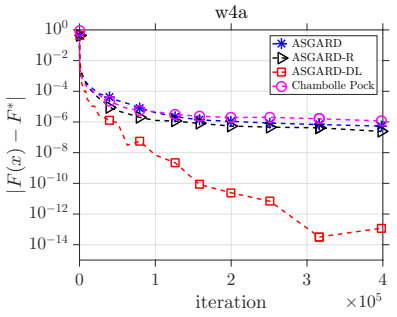

w7a

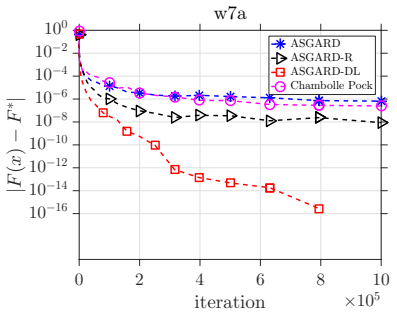

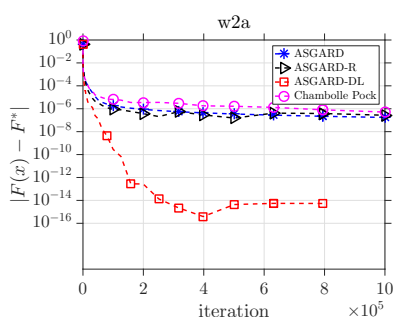

w5a

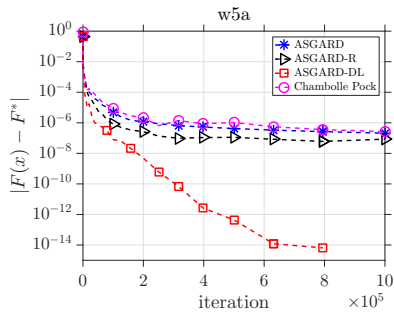

w8a

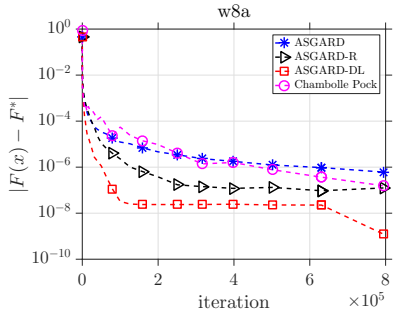

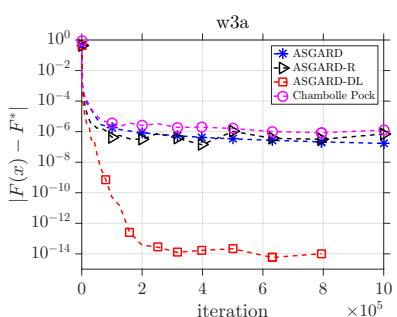

w6a

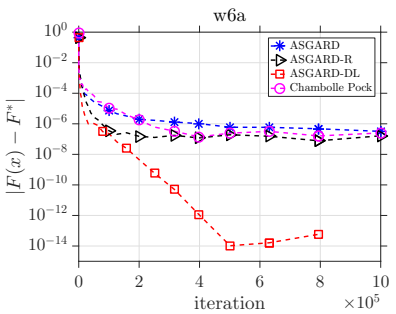

Fig. 5 Performance of 4 algorithms for the $\ell_{1}$-regularized SVM problem on $\{$ w1a, $\cdots$, w8a $\}$.

decreases these parameters to zero, and Chambolle-Pock's method fixes the step-size. Note that the $\mathcal{O}\left(\frac{1}{k}\right)$ rate of Chambolle-Pock's method is achieved via the averaging sequence, which is often much slower than the last iteration as we showed in Figures 5 and 6 .

\subsection{Markowitz Portfolio Optimization}

We consider a classical example from Markowitz portfolio optimization [11. The setting we consider here aims at maximizing the expected return for a given risk level. Assume that we are given a vector $\rho \in \mathbb{R}^{n}$, where $\rho$ is composed 

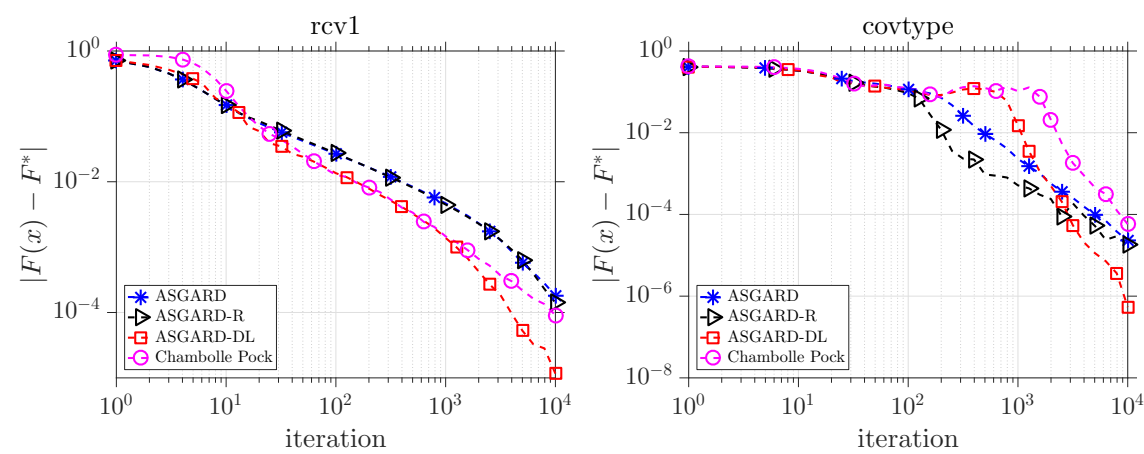

Fig. 6 Performance of 4 algorithms for the $\ell_{1}$-regularized SVM problem on $\{$ rcv1, covtype $\}$.

of expected returns from $n$ assets. This problem can be formulated as

$$
\max _{x \in \mathbb{R}^{p}}\left\{\rho^{\top} x \mid x \in \triangle, \mathbb{E}\left[\left|\left(a_{i}-\rho\right)^{\top} x\right|^{2}\right] \leq \epsilon\right\},
$$

For our setting, we use empirical sample average instead of the expectation and convert the problem to a minimization problem by negating the objective:

$$
\min _{x \in \mathbb{R}^{p}}\left\{-\langle\rho, x\rangle \mid x \in \triangle, \frac{1}{p}\|A x\|_{2}^{2} \leq \epsilon\right\}
$$

where $A=\left[\left(a_{1}-\rho\right),\left(a_{2}-\rho\right), \ldots,\left(a_{n}-\rho\right)\right]^{\top}$. We map this problem to our template (31) by mapping $f(\cdot):=\delta_{\triangle}(\cdot), g(\cdot):=\delta_{\left\{\|\cdot\|_{2} \leq \sqrt{p \epsilon}\right\}}(\cdot)$, and $h(x):=$ $-\langle\rho, x\rangle$. One key step of primal-dual algorithms is computing the projection onto an $\ell_{2}$-norm ball and on a simplex. Here, the complexity of simplex projection is $\mathcal{O}(p \log p)$.

As before, we apply 4 algorithms to solve 38 . We use 4 datasets that are also considered in [6]. The details about the datasets are given in Table 2.

Table 2 Portfolio optimization datasets and parameters of algorithms.

\begin{tabular}{lrrr|rrrrrr}
\hline \multicolumn{4}{c|}{ The size of datasets } & \multicolumn{5}{c}{ Parameters used in 4 algorithms. } \\
\hline Datasets & $n$ & $p$ & $\epsilon$ in $[39)$ & $\beta_{0}$ & RF & $\omega$ & $m_{s}$ & $\tau$ & $\sigma$ \\
\hline DJIA & 507 & 30 & 0.002 & $\|A\|$ & 10 & 1.1 & 11 & $\frac{1}{\|A\|}$ & $\frac{1}{\|A\|}$ \\
NYSE & 5651 & 36 & 0.02 & $100\|A\|$ & 10 & 1.1 & 11 & $\frac{1}{\|A\|}$ & $\frac{1}{\|A\|}$ \\
SP500 & 1276 & 25 & 0.02 & $100\|A\|$ & 10 & 1.2 & 6 & $\frac{1}{\|A\|}$ & $\frac{1}{\|A\|}$ \\
TSE & 1258 & 88 & 0.002 & $100\|A\|$ & 10 & 1.1 & 11 & $\frac{1}{\|A\|}$ & $\frac{1}{\|A\|}$ \\
\hline
\end{tabular}

We summarized the parameters that we used for these algorithms in Table 2, where $\beta_{0}$ is common to ASGARD, ASGARD-restart, our algorithm, restart frequency (RF) is specific to ASGARD-restart, $\omega$ and $m_{s}$ are specific to our algorithm and $\tau$ and $\sigma$ are specific to Chambolle-Pock's algorithm.

We have tested 4 algorithms on 4 real datasets and the results are compiled in Figure 7. As can be seen, except for SP500 dataset, Algorithm2 significantly outperforms the other methods and shows a much faster practical performance 

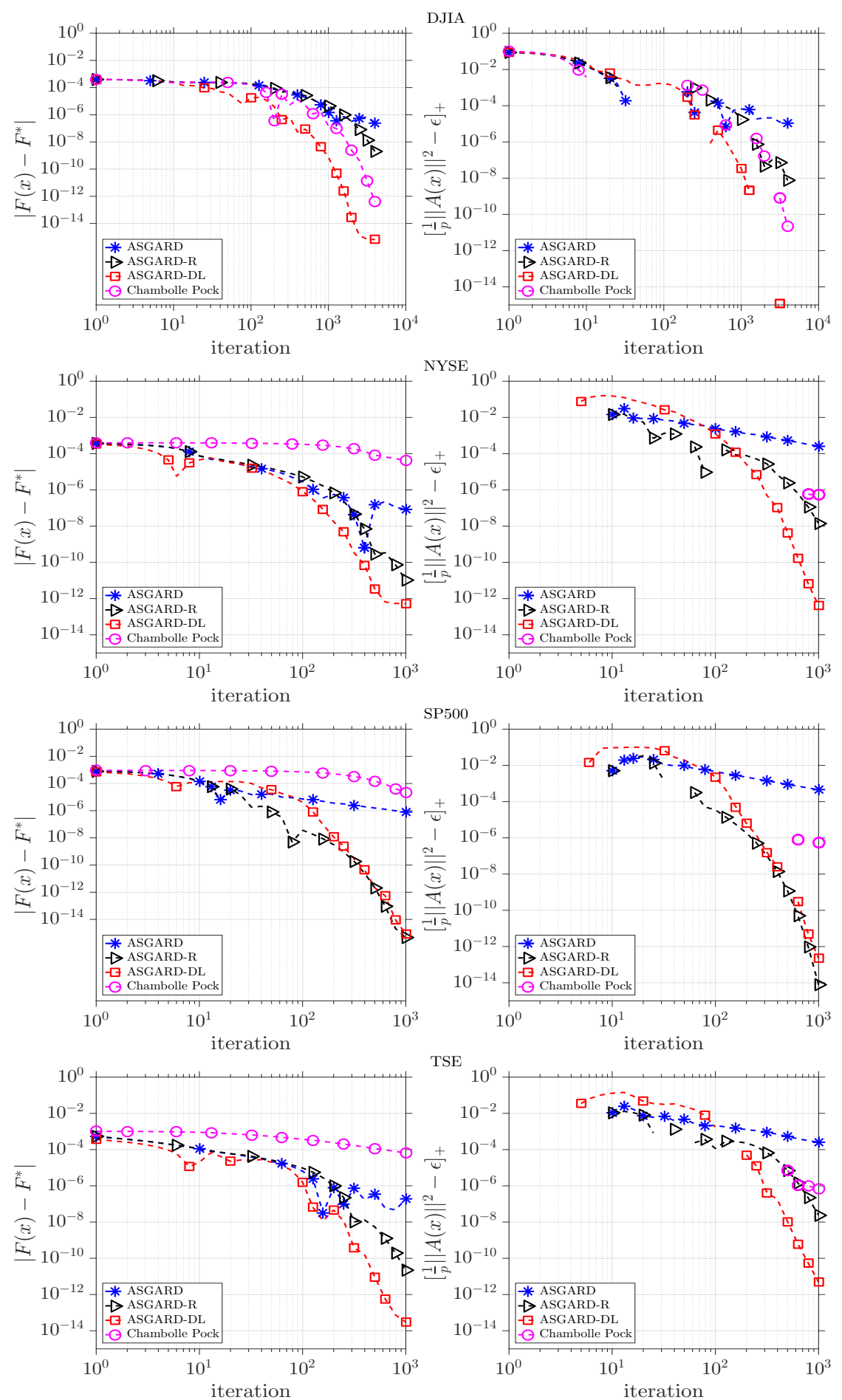

Fig. 7 Performance of 4 algorithms for Markowitz portfolio optimization problem on 4 real datasets. 
than $\mathcal{O}\left(\frac{1}{k}\right)$ guarantee. For SP500 dataset, ASGARD-restart algorithm shows a comparable performance to our method. However, as discussed in [58, the effect of restarting to ASGARD method is not understood theoretically. Our algorithm theoretically preserves the best-known $\mathcal{O}\left(\frac{1}{k}\right)$ guarantee while performing as fast as, and most of the times faster than the heuristic restarting ASGARD method.

\subsection{Sparse Subspace Clustering}

In the last example, we consider the following sparse subspace clustering problem which has broad applications in machine learning, computer vision and image processing. This problem is studied extensively in the literature [26, 25, 53]. In this problem setting, we assume that there exist $n$ points $\left\{x_{1}, x_{2}, \cdots, x_{n}\right\} \in$ $\mathbb{R}^{p}$ lying in the union of subspaces in $\mathbb{R}^{p}$. We form a matrix $X \in \mathbb{R}^{p \times n}$ by stacking $\left\{x_{1}, x_{2}, \cdots, x_{n}\right\}$ as the columns. With this notation, each point can be represented as

$$
x_{j}=X c_{j}+e_{j}, \text { s.t. }\left[c_{j}\right]_{j}=0 \text { and } \mathbb{1}^{\top} c_{j}=1 .
$$

where $c_{j} \in \mathbb{R}^{n}$ represents the coefficients to represent point $x_{j} \in \mathbb{R}^{p}$ as an affine combination of other points, $e_{j} \in \mathbb{R}^{p}$ is the representation error and $\mathbb{1} \in \mathbb{R}^{n}$ is a vector of 1 's.

This formulation can be represented compactly by stacking $c_{j}$ to the $j^{\text {th }}$ column of matrix $C$ as follows:

$$
X=C X \text { s.t. } \operatorname{diag}(C)=0, C^{\top} \mathbb{1}=\mathbb{1} .
$$

The optimization problem that we will tackle in this subsection is referred to as an SSC-Lasso problem in the literature, and is written as

$$
\min _{C \in \mathbb{R}^{n \times n}}\left\{\|C\|_{1}+\frac{\lambda}{2}\|X-C X\|^{2} \mid \operatorname{diag}(C)=0, C^{\top} \mathbb{1}=\mathbb{1}\right\} .
$$

In [25] and [26], ADMM is used to solve (41) and recently, [53] proposed an efficient implementation of ADMM and an application of standard accelerated proximal scheme to this setting. One drawback of applying accelerated proximal schemes to (41) is the evaluation of the proximal operator of an $\ell_{1}$-norm over the linear constraint $C^{\top} \mathbb{1}=\mathbb{1}$. This requires additional computation cost of $\log (n) \mathrm{pn}^{2}$. We fit (41) into our template (31) by defining $f(\cdot):=\|\cdot\|_{1}+\delta_{\{\operatorname{diag}(\cdot)=0\}}(\cdot), g(\cdot):=\delta_{\{\langle\cdot, \mathbb{1}\rangle=\mathbb{1}\}}(\cdot)$, and $h(\cdot)=\frac{\lambda}{2}\|X-X(\cdot)\|^{2}$. If we apply Algorithm 2 to solve this reformulation, then no extra computation cost is incurred as in accelerated proximal gradient methods.

We use a classic benchmark Extended Yale B dataset [30] to test the sparse subspace clustering problem (41). This dataset contains face pictures of 38 individuals taken under 64 different environmental conditions. As previous works, we use downsampled images of size $48 \times 42$ pixels which correspond to $p=2016$. We ran experiments with ADMM, TFOCS, and our method ASGARD-DL. We note that our method includes tuning parameters similar to ADMM. We use $\beta_{0}:=\sqrt{\|M\|}$, where $M(C)=C^{\top} \mathbb{1}$. We randomly selected 
$m=2,3,5$ clusters and ran 3 trials for each case. We have used the implementation of ADMM [26,25] and TFOCS [53] provided by the authors of [53,26. 25. For fair comparison, we ran the algorithms for the same duration of time and reported the results accordingly. We used objective value and clustering error as comparison measures as [53].

Table 3 Comparison of 3 methods on the SSC-Lasso problem with $m=2,3,5$ clusters and 3 independent trials of each.

\begin{tabular}{c|r|r|r}
\hline Problem & ADMM & TFOCS & ASGARD-DL \\
\hline$(n=2)$-objective-trial 1 & 236.5653 & 226.4371 & 225.7578 \\
$(n=2)$-Clustering error-trial 1 & 0.0312 & 0.0391 & 0.0391 \\
\hline$(n=2)$-objective-trial 2 & 200.3710 & 192.2985 & 191.5177 \\
$(n=2)$-Clustering error-trial 2 & 0.0234 & 0.0469 & 0.0469 \\
\hline$(n=2)$-objective-trial 3 & 197.2510 & 188.7655 & 188.1555 \\
$(n=2)$-Clustering error-trial 3 & 0.0703 & 0.0938 & 0.0938 \\
\hline$(n=3)$-objective-trial 1 & 329.9188 & 320.9690 & 319.5887 \\
$(n=3)$-Clustering error-trial 1 & 0.0156 & 0.0156 & 0.0312 \\
\hline$(n=3)$-objective-trial 2 & 341.1980 & 330.6395 & 329.5704 \\
$(n=3)$-Clustering error-trial 2 & 0.0729 & 0.0677 & 0.0677 \\
\hline$(n=3)$-objective-trial 3 & 398.8778 & 389.3963 & 388.0739 \\
$(n=3)$-Clustering error-trial 3 & 0.4375 & 0.3594 & 0.3646 \\
\hline$(n=5)$-objective-trial 1 & 549.8250 & 530.0340 & 526.1905 \\
$(n=5)$-Clustering error-trial 1 & 0.1625 & 0.1156 & 0.0906 \\
\hline$(n=5)$-objective-trial 2 & 482.8483 & 467.0535 & 461.6563 \\
$(n=5)$-Clustering error-trial 2 & 0.2188 & 0.1125 & 0.1562 \\
\hline$(n=5)$-objective-trial 3 & 1029.5459 & 1017.7089 & 1025.6752 \\
$(n=5)$-Clustering error-trial 3 & 0.3156 & 0.3469 & 0.3156 \\
\hline
\end{tabular}

We can see from Table 3 that our method consistently outperforms other methods in terms of objective values, and has similar performance in terms of the clustering error. We present our algorithm as another candidate for solving the classical sparse subspace clustering problem with a lower per iteration cost than previous approaches ADMM and TFOCS and similar performance.

\section{Further discussion and comparison with previous work}

Theory and numerical methods for solving (1) and (3) are well-studied in the literature. Due to such a large proportion of solution methods, we only focus on some recent works that are the most related to our method developed in this paper. We briefly survey these results to highlight the similarities and differences with our work.

In [47, Nesterov proposed combining smoothing technique and accelerated gradient methods to obtain $\mathcal{O}\left(\frac{1}{\varepsilon}\right)$-iteration complexity to obtain an $\varepsilon$ approximate solution to (1). However, this method requires $\varepsilon$ to be predefined, and both primal and dual domains are bounded. In addition, the step-size of the underlying gradient-type scheme is proportional to $\varepsilon$, which is often small. 
This leads to a poor performance in early iterations. In [46], Nesterov introduced an excessive gap technique to develop new algorithms that allow the smoothness parameter to be adaptively updated. Nevertheless, these methods still require both primal and dual domains to be bounded, and one additional proximal operator for every two iterations.

In 13, 14, A. Chambolle and T. Pock proposed a primal-dual algorithm to solve (1) that achieves $\mathcal{O}\left(\frac{1}{k}\right)$-convergence rate. This rate is guaranteed on a gap function and also requires both primal and dual domains to be bounded, which is unfortunately not applicable to (3). In addition, the guarantee of their methods relies on ergodic or weighted averaging sequences. Note that, in sparse and low-rank optimization and image processing, taking averaging sequence unfortunately destroys desired structures of approximate solutions. In addition, as also presented with numerical evidence, averaging sequences perform poorly in practice.

In 69, the authors proposed a homotopy algorithm called Homotopy Smoothing algorithm (HOPS) which also essentially relies on Nesterov's smoothing technique [7]. HOPS employs a similar strategy to ours in the sense of having a double loop structure. However, this method suffers from several drawbacks. First, it only applies to unconstrained problems as in (1), but not to (3) due to the unboundedness of the dual domain. Second, it requires knowing $\varepsilon_{0}=P\left(x^{0}\right)-P\left(x^{\star}\right)$ to be able to set the initial smoothness parameter. Third, HOPS requires tuning the number of inner iterations and the rate at which the smoothness parameter is going to be reduced. The alternative of HOPS to alleviate this issue requires a bounded primal domain which further restricts the usage of their method.

For constrained problem (3), among different methods, augmented Lagrangian (ALM), alternating direction method of multipliers (ADMM), alternating minimization algorithms (AMA), and penalty methods are the most popular. Inexact augmented Lagrangian methods (iALM) [36, 43, 68, relies on a double loop structure similar to our method. However, termination rules for these methods require the desired accuracy $\varepsilon$ to be set a priori. In addition, in practice, it is not easy to check when the inner problem is solved to an $\varepsilon_{k}$-accuracy in the $k$-th iteration. Such an estimate is often derived from the worst-case complexity bound of the underlying solution method, and therefore, the corresponding algorithm is not efficient in practice.

While ADMM works really well and is widely used in practice, AMA is rarely used and requires additional conditions to converge. The best-known convergence rate of ADMM and its variants such as linearized ADMM and preconditioned ADMM is $\mathcal{O}\left(\frac{1}{k}\right)$ under standard assumptions [32, 39, 40, 50, 67. Moreover, this rate is given in an ergodic sense, and examples show that such a rate is optimal. See [10 for more information about the behavior of ADMM. In practice, however, the ergodic rate is rather pessimistic, which is much slower than the last iterate sequence (see Subsection 4.1 as an example). So far, we are not aware of any work showing an $\mathcal{O}\left(\frac{1}{k}\right)$-rate of the standard $\mathrm{ADMM}$ or its linearized and preconditioned ADMM in the last iterate. A 


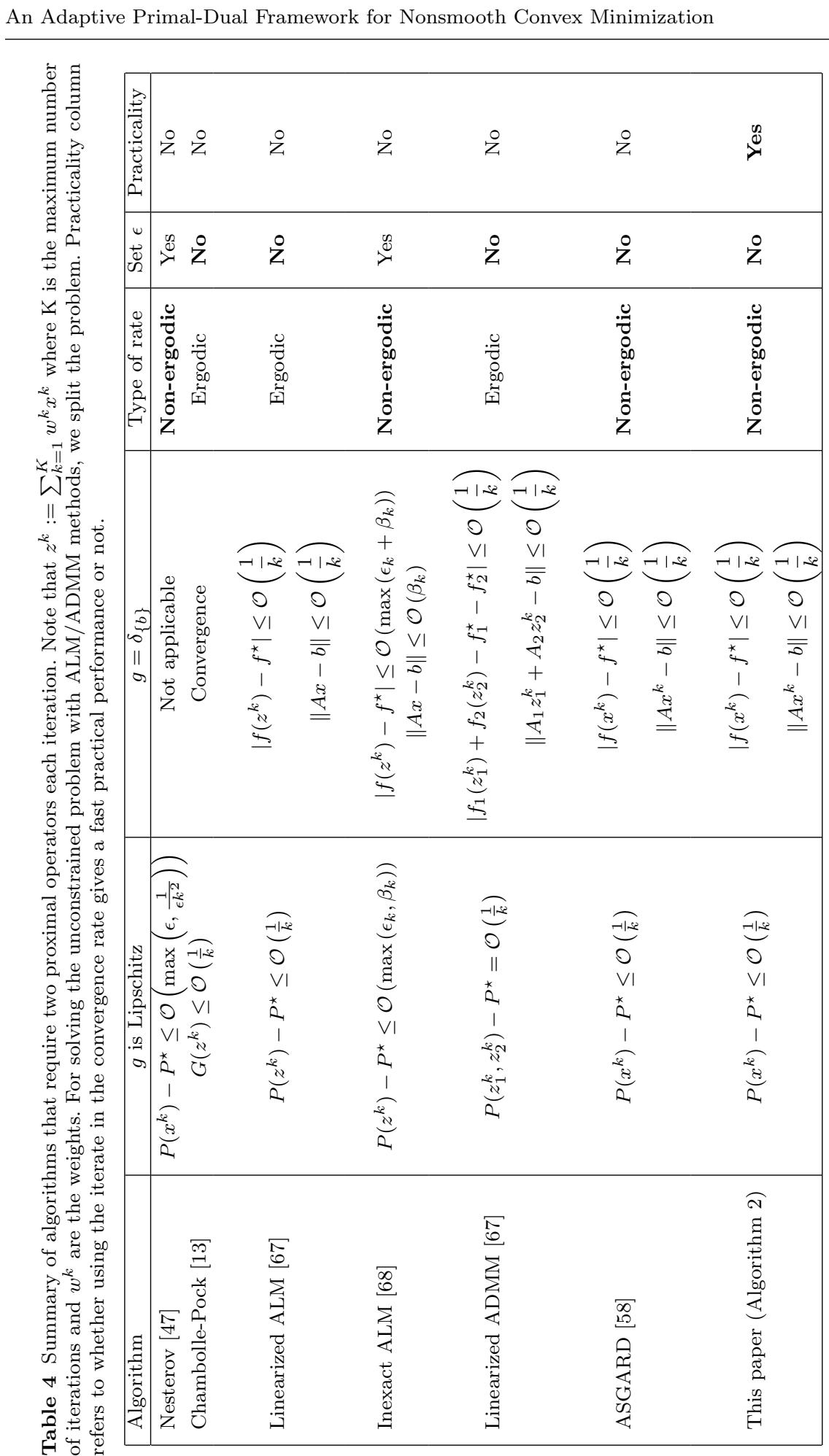


recent work [37] combined preconditioned/linearized ADMM and Nesterov's accelerated schemes to achieve an $\mathcal{O}\left(\frac{1}{k}\right)$-non-ergodic convergence rate.

Penalty methods use a quadratic penalty term to move the constraints to the objective and solve the subproblems by changing the penalty parameter [35,42. Similar to iALM, these methods also do not have clear implementable termination rules for the inner loop. In addition, they do not involve dual variables. Therefore, they are often less competitive with primal-dual methods. A recent work [57] proposed a new alternating quadratic penalty algorithm to solve (3) that has the same $\mathcal{O}\left(\frac{1}{k}\right)$-non-ergodic convergence rate as in this paper. Nevertheless, this method is completely different from this paper and does not have an update on the dual center.

Compared to our previous work [58, ASGARD, our new algorithm shares some similarities but also has several differences. First, it has inner and outer loops but the guarantee is on the overall iterations. Second, it works with any Bregman divergence induced by a general prox-function when solving (1), while ASGARD only works with the Bregman distances induced by a strongly convex and Lipschitz gradient prox-function. This excludes some important Bregman divergences such as the Kullback-Leibler (KL) divergence. Third, our algorithm allows us to use different norms while computing proximal operators, compared to ASGARD which works with only Euclidean norms. Fourth, it automatically restarts both the primal and dual variables as well as the parameters. It also has a rigorous convergence guarantee, while the practical restarting variant of ASGARD does not have convergence guarantee.

We developed a novel analysis for our double loop structured smoothing algorithm which allowed us to derive flexible rules for parameters in both unconstrained and constrained problems, in contrast to [69. Our analysis gives insights on the heuristic restarting strategies in 58 as well as on the number of inner iterations in the algorithm. It also gives explicit number of iterations for the inner subproblems and does not require to predefine the horizon as opposed to iALM. Table 5 summarizes the key differences between different methods we have discussed in this paper.

Table 5 A comparison with previous work ( $\beta$ is a smoothness parameter defined in 10$)$.

\begin{tabular}{l|l|l}
\hline \multicolumn{1}{c|}{ ADMM/iALM } & Penalty / HOPS / ASGARD & \multicolumn{1}{c}{ This work } \\
\hline Constant or adaptive $\beta$. & Analytically drive $\beta$ to 0. & Analytically drive $\beta$ to 0. \\
\hline Update the dual center. & $\begin{array}{l}\text { Do not move the dual } \\
\text { center. }\end{array}$ & Update the dual center. \\
\hline $\begin{array}{l}\text { Theory is driven by the } \\
\text { convergence in the dual. }\end{array}$ & $\begin{array}{l}\text { Do not analyze the con- } \\
\text { vergence of the dual. }\end{array}$ & $\begin{array}{l}\text { Only analyze the stabil- } \\
\text { ity of the primal-dual se- } \\
\text { quence. }\end{array}$ \\
\hline $\begin{array}{l}\text { Inner problems are solved } \\
\text { inexactly. }\end{array}$ & $\begin{array}{l}\text { Inner problems are solved } \\
\text { inexactly. }\end{array}$ & $\begin{array}{l}\text { Only ensure stability for } \\
\text { the number of inner itera- } \\
\text { tions and smoothness pa- } \\
\text { rameter. }\end{array}$ \\
\hline
\end{tabular}


6 Convergence analysis: The proof of Theorems 3.1 and 3.2

We present the full proof of Theorems 3.1 and 3.2 in this section.

\subsection{The proof of Theorem 3.1; Convergence of Algorithm 2 for (1)}

With the same argument as in [58, Lemma 11], we can prove the following estimate at the $k$-th iteration at the state $s$ of the outer loop, i.e., $K_{s} \leq k<$ $K_{s+1}:=K_{s}+m_{s}$, of Algorithm 2 .

$$
\begin{aligned}
& S_{\beta_{s}}\left(\bar{x}^{k+1} ; \dot{y}^{s}\right)+\frac{\tau_{k}^{2}\|A\|^{2}}{\beta_{s}} d_{\mathcal{X}}\left(x^{\star}, \hat{x}^{k+1}\right) \leq\left(1-\tau_{k}\right) S_{\beta_{s}}\left(\bar{x}^{k} ; \dot{y}^{s}\right)+\frac{\tau_{k}^{2}\|A\|^{2}}{\beta_{s}} d \mathcal{X}\left(x^{\star}, \hat{x}^{k}\right) \\
& -\tau_{k} \beta_{s} b_{\mathcal{Y}}\left(y_{\beta_{s}}^{*}\left(A \tilde{x}^{k} ; \dot{y}^{s}\right), \dot{y}^{s}\right)-\frac{\left(1-\tau_{k}\right) \beta_{s}}{2}\left\|y_{\beta_{s}}^{*}\left(A \tilde{x}^{k} ; \dot{y}^{s}\right)-y_{\beta_{s}}^{*}\left(A \bar{x}^{k} ; \dot{y}^{s}\right)\right\|_{\mathcal{Y}}^{2},
\end{aligned}
$$

where $S_{\beta}(\bar{x} ; \dot{y}):=P_{\beta}(\bar{x} ; \dot{y})-P\left(x^{\star}\right)$. Note that this estimate remains true if we use APG instead of FISTA, and APG with Option 2.

Next, by strong convexity of $b_{\mathcal{Y}}(\cdot, \dot{y})$, the optimality condition of $g_{\beta}$-subproblem and convexity of $g^{*}(\cdot)$, we have

$$
\begin{aligned}
g_{\beta}(A \bar{x} ; \dot{y}) & =\max _{y \in \mathbb{R}^{n}}\left\{\langle A \bar{x}, y\rangle-g^{*}(y)-\beta b \mathcal{Y}(y, \dot{y})\right\} \\
& \geq\left\langle A \bar{x}, y^{\star}\right\rangle-g^{*}\left(y^{\star}\right)-\beta b \mathcal{Y}\left(y^{\star}, \dot{y}\right)+\beta b \mathcal{Y}\left(y^{\star}, y_{\beta}^{*}(A \bar{x} ; \dot{y})\right) .
\end{aligned}
$$

Now, from the optimality condition of (1), we have $-A^{\top} y^{\star} \in \partial f\left(x^{\star}\right)$. Using this inclusion and convexity of $f$, we can derive

$$
f(\bar{x}) \geq f\left(x^{\star}\right)+\left\langle-A^{\top} y^{\star}, \bar{x}-x^{\star}\right\rangle .
$$

Combining 43 and 44 , we get

$$
\begin{aligned}
S_{\beta}(\bar{x} ; \dot{y}) & =P_{\beta}(\bar{x} ; \dot{y})-P\left(x^{\star}\right)=f(\bar{x})+g_{\beta}(A \bar{x} ; \dot{y})-\left(f\left(x^{\star}\right)+g\left(A x^{\star}\right)\right) \\
& \geq-\beta b \mathcal{Y}\left(y^{\star}, \dot{y}\right)+\beta b \mathcal{Y}\left(y^{\star}, y_{\beta}^{*}(A \bar{x} ; \dot{y})\right) .
\end{aligned}
$$

From 42 , if we ignore the two last terms, which are nonpositive, then for $K_{s} \leq k \leq K_{s}+m_{s}-1$ we obtain

$$
\frac{1}{\tau_{k}^{2}} S_{\beta_{s}}\left(\bar{x}^{k+1} ; \dot{y}^{s}\right)+\frac{\|A\|^{2}}{\beta_{s}} d_{\mathcal{X}}\left(x^{\star}, \hat{x}^{k+1}\right) \leq \frac{1-\tau_{k}}{\tau_{k}^{2}} S_{\beta_{s}}\left(\bar{x}^{k} ; \dot{y}^{s}\right)+\frac{\|A\|^{2}}{\beta_{s}} d_{\mathcal{X}}\left(x^{\star}, \hat{x}^{k}\right) .
$$

Let us define $D_{k}^{s}:=S_{\beta_{s}}\left(\bar{x}^{k} ; \dot{y}^{s}\right)+\beta_{s} D_{\mathcal{Y}} \geq P\left(\bar{x}^{k}\right)-P\left(x^{\star}\right) \geq 0$. By adding $\frac{1}{\tau_{k}^{2}} \beta_{s} D_{\mathcal{Y}}$ to both sides of (46) and using the definition of $D_{k}^{s}$, we obtain

$$
\frac{1}{\tau_{k}^{2}} D_{k+1}^{s}+\frac{\|A\|^{2}}{\beta_{s}} d_{\mathcal{X}}\left(x^{\star}, \hat{x}^{k+1}\right) \leq \frac{\left(1-\tau_{k}\right)}{\tau_{k}^{2}} D_{k}^{s}+\frac{\|A\|^{2}}{\beta_{s}} d_{\mathcal{X}}\left(x^{\star}, \hat{x}^{k}\right)+\frac{\beta_{s}}{\tau_{k}} D_{\mathcal{Y}} .
$$

Let us choose $\tau_{k}=\frac{2}{k-K_{s}+2}$. Then, it is clear that $\tau_{K_{s}}=1$. Moreover, $\frac{1-\tau_{k}}{\tau_{k}^{2}}=$ $\frac{\left(k-K_{s}+2\right)\left(k-K_{s}\right)}{4} \leq \frac{\left(k-K_{s}+1\right)^{2}}{4}=\frac{1}{\tau_{k-1}^{2}}$. In this case, we can overestimate 47) as

$$
\frac{1}{\tau_{k}^{2}} D_{k+1}^{s}+\frac{\|A\|^{2}}{\beta_{s}} d_{\mathcal{X}}\left(x^{\star}, \hat{x}^{k+1}\right) \leq \frac{1}{\tau_{k-1}^{2}} D_{k}^{s}+\frac{\|A\|^{2}}{\beta_{s}} d \mathcal{X}\left(x^{\star}, \hat{x}^{k}\right)+\frac{\beta_{s}}{\tau_{k}} D_{\mathcal{Y}} .
$$


Taking a telescope from $k=K_{s}+1$ to $k=K_{s+1}-1=K_{s}+m_{s}-1$ of 48 and reuse (47) for $k=K_{s}$, we obtain

$$
\begin{aligned}
D_{K_{s+1}}^{s} & +\frac{\tau_{K_{s+1}-1}^{2}\|A\|^{2}}{\beta_{s}} d \mathcal{X}\left(x^{\star}, \hat{x}^{K_{s+1}}\right) \leq \frac{\tau_{K_{s+1}-1}^{2}\left(1-\tau_{K_{s}}\right)}{\tau_{K_{s}}^{2}} D_{K_{s}}^{s} \\
& +\frac{\tau_{K_{s+1}-1}^{2}\|A\|^{2}}{\beta_{s}} d_{\mathcal{X}}\left(x^{\star}, \hat{x}^{K_{s}}\right)+\beta_{s} \tau_{K_{s+1}-1}^{2} D \mathcal{Y} \sum_{j=K_{s}}^{K_{s}+m_{s}-1} \frac{1}{\tau_{j}} \\
& \stackrel{(i)}{\leq} \frac{\tau_{K_{s+1}-1}^{2}\|A\|^{2}}{\beta_{s}} d_{\mathcal{X}}\left(x^{\star}, \hat{x}^{K_{s}}\right)+\beta_{s} \tau_{K_{s+1}-1}^{2} D_{\mathcal{Y}} \sum_{j=K_{s}}^{K_{s}+m_{s}-1} \frac{1}{\tau_{j}},
\end{aligned}
$$

where $(i)$ holds since $\tau_{K_{s}}=1$. Since $\tau_{k}=\frac{2}{k-K_{s}+2}$, we have $\tau_{K_{s+1}-1}=\frac{2}{m_{s}+1}$ and $\sum_{j=K_{s}}^{K_{s}+m_{s}-1} \frac{1}{\tau_{j}}=\frac{m_{s}\left(m_{s}+3\right)}{4}$. Using this relation, the last estimate leads to

$$
D_{K_{s+1}}^{s}+\frac{4\|A\|^{2}}{\left(m_{s}+1\right)^{2} \beta_{s}} d_{\mathcal{X}}\left(x^{\star}, \hat{x}^{K_{s+1}}\right) \leq \frac{4\|A\|^{2}}{\left(m_{s}+1\right)^{2} \beta_{s}} d_{\mathcal{X}}\left(x^{\star}, \hat{x}^{K_{s}}\right)+\frac{\beta_{s} m_{s}\left(m_{s}+3\right)}{\left(m_{s}+1\right)^{2}} D_{\mathcal{Y}}
$$

Since $D_{K_{s+1}}^{s}=S_{\beta_{s}}\left(\bar{x}^{K_{s+1}} ; \dot{y}^{s}\right)+\beta_{s} D_{\mathcal{Y}}$, the last estimate leads to

$$
\begin{aligned}
S_{\beta_{s}}\left(\bar{x}^{K_{s+1}} ; \dot{y}^{s}\right)+\frac{4\|A\|^{2}}{\left(m_{s}+1\right)^{2} \beta_{s}} d_{\mathcal{X}}\left(x^{\star}, \hat{x}^{K_{s+1}}\right) & \leq \frac{4\|A\|^{2}}{\left(m_{s}+1\right)^{2} \beta_{s}} d_{\mathcal{X}}\left(x^{\star}, \hat{x}^{K_{s}}\right) \\
& +\frac{\beta_{s}\left(m_{s}-1\right)}{\left(m_{s}+1\right)^{2}} D \mathcal{Y} .
\end{aligned}
$$

Here, we note that $\frac{\beta_{s} m_{s}\left(m_{s}+3\right)}{\left(m_{s}+1\right)^{2}} D_{\mathcal{Y}}-\beta_{s} D_{\mathcal{Y}}=\frac{\beta_{s}\left(m_{s}-1\right)}{\left(m_{s}+1\right)^{2}} D_{\mathcal{Y}}$.

Next, from (45), we have

$$
S_{\beta_{s}}\left(\bar{x}^{K_{s+1}} ; \dot{y}^{s}\right) \geq \beta_{s} b_{\mathcal{Y}}\left(y^{\star}, y_{\beta_{s}}^{*}\left(A \bar{x}^{K_{s+1}} ; \dot{y}^{s}\right)\right)-\beta_{s} b_{\mathcal{Y}}\left(y^{\star}, \dot{y}^{s}\right) .
$$

Then, combining 49 and 50 with the fact that $\dot{y}^{s+1} \leftarrow y_{\beta_{s}}^{*}\left(A \bar{x}^{K_{s+1}} ; \dot{y}^{s}\right)$, we can show that

$$
\begin{aligned}
\frac{4\|A\|^{2}}{\left(m_{s}+1\right)^{2}} d_{\mathcal{X}}\left(x^{\star}, \hat{x}^{K_{s+1}}\right)+\beta_{s}^{2} b_{\mathcal{Y}}\left(y^{\star}, \dot{y}^{s+1}\right) & \leq \frac{4\|A\|^{2}}{\left(m_{s}+1\right)^{2}} d_{\mathcal{X}}\left(x^{\star}, \hat{x}^{K_{s}}\right) \\
& +\beta_{s}^{2} b_{\mathcal{Y}}\left(y^{\star}, \dot{y}^{s}\right)+\frac{\beta_{s}^{2}\left(m_{s}-1\right)}{\left(m_{s}+1\right)^{2}} D_{\mathcal{Y}} .
\end{aligned}
$$

Using the update rule (18) $m_{s+1} \leftarrow\left\lfloor\omega\left(m_{s}+1\right)+1\right\rfloor-1$, we have

$$
\omega\left(m_{s}+1\right) \leq m_{s+1}+1 \leq \omega\left(m_{s}+1\right)+1 .
$$

Define $q_{s}:=\frac{\beta_{s}^{2}\left(m_{s}-1\right)}{\left(m_{s}+1\right)^{2}} D \mathcal{Y}$, then using $\beta_{s+1} \leftarrow \frac{\beta_{s}}{\omega}$ from 18$)$ and $\omega\left(m_{s}+1\right) \leq$ $m_{s+1}+1$, we obtain

$$
\begin{aligned}
\frac{4\|A\|^{2}}{\left(m_{s+1}+1\right)^{2}} d \mathcal{X}\left(x^{\star}, \hat{x}^{K_{s+1}}\right)+\beta_{s+1}^{2} b_{\mathcal{Y}}\left(y^{\star}, \dot{y}^{s+1}\right) & \leq \frac{1}{\omega^{2}}\left[\frac{4\|A\|^{2}}{\left(m_{s}+1\right)^{2}} d \mathcal{X}\left(x^{\star}, \hat{x}^{K_{s}}\right)\right. \\
& \left.+\beta_{s}^{2} b_{\mathcal{Y}}\left(y^{\star}, \dot{y}^{s}\right)\right]+\frac{q_{s}}{\omega^{2}}
\end{aligned}
$$

Telescoping this inequality from $s \leftarrow 0$ to $s \leftarrow s-1$, we finally obtain

$$
\begin{aligned}
\frac{4\|A\|^{2}}{\left(m_{s}+1\right)^{2}} d_{\mathcal{X}}\left(x^{\star}, \hat{x}^{K_{s}}\right)+\beta_{s}^{2} b_{\mathcal{Y}}\left(y^{\star}, \dot{y}^{s}\right) & \leq \frac{1}{\omega^{2 s}}\left[\frac{4\|A\|^{2}}{\left(m_{0}+1\right)^{2}} d_{\mathcal{X}}\left(x^{\star}, \hat{x}^{0}\right)\right. \\
& \left.+\beta_{0}^{2} b_{\mathcal{Y}}\left(y^{\star}, \dot{y}^{0}\right)\right]+Q_{s}
\end{aligned}
$$


where $Q_{s}:=\frac{q_{s-1}}{\omega^{2}}+\frac{q_{s-2}}{\omega^{4}}+\cdots+\frac{q_{0}}{\omega^{2 s}}$. If we ignore the second term on the left-hand side, which is nonnegative, and use the equality $\hat{x}^{0}=\bar{x}^{0}$, we obtain

$$
\frac{4\|A\|^{2}}{\left(m_{s}+1\right)^{2}} d_{\mathcal{X}}\left(x^{\star}, \hat{x}^{K_{s}}\right) \leq \frac{1}{\omega^{2 s}}\left[\frac{4\|A\|^{2}}{\left(m_{0}+1\right)^{2}} d_{\mathcal{X}}\left(x^{\star}, \bar{x}^{0}\right)+\beta_{0}^{2} b_{\mathcal{Y}}\left(y^{\star}, \dot{y}^{0}\right)\right]+Q_{s}
$$

Lower bounding the second term on the left-hand side in 49 by 0 , and combining the result with 53 we obtain

$$
\begin{aligned}
S_{\beta_{s}}\left(\bar{x}^{K_{s+1}} ; \dot{y}^{s}\right) & \leq \frac{4\|A\|^{2}}{\left(m_{s}+1\right)^{2} \beta_{s}} d_{\mathcal{X}}\left(x^{\star}, \hat{x}^{K_{s}}\right)+\frac{\beta_{s}\left(m_{s}-1\right)}{\left(m_{s}+1\right)^{2}} D_{\mathcal{Y}} \\
& \leq \frac{1}{\beta_{s} \omega^{2 s}}\left[\frac{4\|A\|^{2}}{\left(m_{0}+1\right)^{2}} d_{\mathcal{X}}\left(x^{\star}, \bar{x}^{0}\right)+\beta_{0}^{2} b_{\mathcal{Y}}\left(y^{\star}, \dot{y}^{0}\right)\right]+\overbrace{\frac{Q_{s}}{\beta_{s}}+\frac{q_{s}}{\beta_{s}}}^{\hat{Q}_{s}} .
\end{aligned}
$$

Note from (18) that $\beta_{s}=\frac{\beta_{0}}{\omega^{s}}$ implies $\frac{1}{\beta_{s} \omega^{2 s}}=\frac{1}{\beta_{0} \omega^{s}}$. By induction of (51), yields

$$
m_{0} \omega^{s}<\left(m_{0}+1\right) \omega^{s}-1 \leq m_{s} \leq\left(m_{0}+\frac{\omega}{\omega-1}\right) \omega^{s}-\frac{\omega}{\omega-1}<\kappa_{0} \omega^{s}
$$

where $\kappa_{0}:=m_{0}+\frac{\omega}{\omega-1}>0$ for $\omega>1$ and $m_{0} \geq 1$. Using these bounds, one has

$$
q_{s}=\frac{\beta_{s}^{2}\left(m_{s}-1\right)}{\left(m_{s}+1\right)^{2}} D_{\mathcal{Y}} \leq \frac{\beta_{0}^{2}}{\omega^{2 s}\left(m_{s}+1\right)} D_{\mathcal{Y}} \leq \frac{\beta_{0}^{2}}{m_{0} \omega^{3 s}} D \mathcal{Y}
$$

Substituting this inequality into $\hat{Q}_{s}$, we can bound

$$
\begin{aligned}
\hat{Q}_{s} & :=\frac{1}{\beta_{s}}\left(q_{s}+\frac{q_{s-1}}{\omega^{2}}+\cdots+\frac{q_{0}}{\omega^{2 s}}\right) \leq \frac{\beta_{0} \omega^{s} D_{\mathcal{Y}}}{m_{0}}\left(\frac{1}{\omega^{3 s}}+\frac{1}{\omega^{2} \omega^{3(s-1)}}+\cdots+\frac{1}{\omega^{2 s}}\right) \\
& \leq \frac{\beta_{0} D \mathcal{y}}{m_{0} \omega^{s}}\left(\frac{1}{\omega^{s}}+\frac{1}{\omega^{s-1}}+\cdots+\frac{1}{\omega}+1\right) \leq \frac{\beta_{0} \omega D \mathcal{y}}{(\omega-1) m_{0} \omega^{s}}
\end{aligned}
$$

Using $m_{s} \leq \kappa_{0} \omega^{s}$ in 55 to estimate the total number of iterations $K_{s+1}$ as

$$
K_{s+1}=\sum_{i=0}^{s} m_{i} \leq \kappa_{0} \sum_{i=0}^{s} \omega^{i}=\kappa_{0}\left(\frac{\omega^{s+1}-1}{\omega-1}\right) .
$$

This condition leads to $\omega^{s} \geq \frac{(\omega-1) K_{s+1}+\kappa_{0}}{\omega \kappa_{0}}$. Using this estimate, $\beta_{s}=\frac{\beta_{0}}{\omega^{s}}$ and (56) into 54, we obtain

$$
S_{\beta_{s}}\left(\bar{x}^{K_{s+1}} ; \dot{y}^{s}\right) \leq \frac{1}{\beta_{0} \omega^{s}}\left[\frac{4\|A\|^{2}}{\left(m_{0}+1\right)^{2}} d_{\mathcal{X}}\left(x^{\star}, \bar{x}^{0}\right)+\beta_{0}^{2} b_{\mathcal{Y}}\left(y^{\star}, \dot{y}^{0}\right)+\frac{\beta_{0}^{2} \omega D_{\mathcal{Y}}}{(\omega-1) m_{0}}\right] .
$$

Finally, by the fact that $P\left(\bar{x}^{K_{s+1}}\right)-P^{\star} \leq S_{\beta_{s}}\left(\bar{x}^{K_{s+1}} ; \dot{y}^{s}\right)+\beta_{s} D_{\mathcal{Y}}$, and $\beta_{s}=$ $\frac{\beta_{0}}{\omega^{s}} \leq \frac{\omega \beta_{0} \kappa_{0}}{(\omega-1) K_{s+1}+\kappa_{0}}$, the last estimate implies 19 . 
6.2 The proof of Theorem 3.2 Convergence of Algorithm 2 for (3)

By Lemma 3.1, we have $\beta b y\left(y^{\star}, \dot{y}\right)+S_{\beta}(\bar{x} ; \dot{y}) \geq 0$. Let us define $\hat{D}_{k}^{s}:=$ $S_{\beta_{s}}\left(\bar{x}^{k} ; \dot{y}^{s}\right)+\beta_{s} b y\left(y^{\star}, \dot{y}^{s}\right)$. Then, using the same proof as (47) in Theorem 3.1 by replacing $D_{\mathcal{Y}}$ with $b_{\mathcal{Y}}\left(y^{\star}, \dot{y}^{s}\right)$.

$\frac{1}{\tau_{k}^{2}} \hat{D}_{k+1}^{s}+\frac{\|A\|^{2}}{\beta_{s}} d_{\mathcal{X}}\left(x^{\star}, \hat{x}^{k+1}\right) \leq \frac{\left(1-\tau_{k}\right)}{\tau_{k}^{2}} \hat{D}_{k}^{s}+\frac{\|A\|^{2}}{\beta_{s}} d_{\mathcal{X}}\left(x^{\star}, \hat{x}^{k}\right)+\frac{\beta_{s}}{\tau_{k}} b_{\mathcal{Y}}\left(y^{\star}, \dot{y}^{s}\right)$.

From this estimate, with the same proof as (49), we obtain

$$
\begin{aligned}
S_{\beta_{s}}\left(\bar{x}^{K_{s+1}} ; \dot{y}^{s}\right)+\frac{4\|A\|^{2}}{\left(m_{s}+1\right)^{2} \beta_{s}} d_{\mathcal{X}}\left(x^{\star}, \hat{x}^{K_{s+1}}\right) & \leq \frac{4\|A\|^{2}}{\left(m_{s}+1\right)^{2} \beta_{s}} d_{\mathcal{X}}\left(x^{\star}, \hat{x}^{K_{s}}\right) \\
& +\frac{\beta_{s}\left(m_{s}-1\right)}{\left(m_{s}+1\right)^{2}} b_{\mathcal{Y}}\left(y^{\star}, \dot{y}^{s}\right) .
\end{aligned}
$$

Combining this, [50], and the fact that $\dot{y}^{s+1} \leftarrow y_{\beta_{s}}^{*}\left(A \bar{x}^{K_{s+1}} ; \dot{y}^{s}\right)$, we obtain

$$
\begin{aligned}
\frac{4\|A\|^{2}}{\left(m_{s}+1\right)^{2}} d_{\mathcal{X}}\left(x^{\star}, \hat{x}^{K_{s+1}}\right)+\beta_{s}^{2} b_{\mathcal{Y}}\left(y^{\star}, \dot{y}^{s+1}\right) & \leq \frac{4\|A\|^{2}}{\left(m_{s}+1\right)^{2}} d_{\mathcal{X}}\left(x^{\star}, \hat{x}^{K_{s}}\right) \\
& +\frac{\beta_{s}^{2} m_{s}\left(m_{s}+3\right)}{\left(m_{s}+1\right)^{2}} b_{\mathcal{Y}}\left(y^{\star}, \dot{y}^{s}\right) .
\end{aligned}
$$

Let us choose $m_{s+1}:=\left\lfloor\omega\left(m_{s}+1\right)+1\right\rfloor-1$ and $\beta_{s+1}:=\frac{\beta_{s}\left(m_{s+1}+1\right)}{\omega \sqrt{m_{s+1}\left(m_{s+1}+3\right)}}$ as in 29). Then, similar to the proof of (55), we have

$$
m_{0} \omega^{s} \leq m_{s} \leq \kappa_{0} \omega^{s}, \quad \text { and } \quad \beta_{s+1} \leq \frac{\beta_{s}}{\omega} \leq \frac{\beta_{0}}{\omega^{s+1}},
$$

where $\kappa_{0}:=m_{0}+\frac{\omega}{\omega-1}>0$.

Next, we need to lower bound $\beta_{s}$. We can show that, for $m_{s} \geq 1$, we have

$$
\frac{m_{s+1}+1}{\sqrt{m_{s+1}\left(m_{s+1}+3\right)}} \geq 1-\frac{1}{m_{s+1}} \geq 0 \text {. }
$$

In this case, we can estimate $\beta_{s+1}=\frac{\beta_{s}\left(m_{s+1}+1\right)}{\omega \sqrt{m_{s+1}\left(m_{s+1}+3\right)}} \geq \frac{\beta_{s}}{\omega}\left(1-\frac{1}{m_{s+1}}\right)=$ $\frac{\beta_{s}}{\omega}-\frac{\beta_{s}}{m_{s+1} \omega}$. Substituting 60 on $m_{s+1}$ and $\beta_{s}$ into this inequality, we obtain

$$
\beta_{s+1} \geq \frac{\beta_{s}}{\omega}-\frac{c_{0}}{\omega^{2 s+1}}, \quad \text { where } \quad c_{0}:=\frac{\beta_{0}}{\omega m_{0}} .
$$

This condition leads to $\omega \beta_{s+1}+\frac{c_{0}}{\omega^{2 s}} \geq \beta_{s}$. By induction, we can show that $\omega^{s} \beta_{s}+c_{0} \sum_{j=0}^{s-1} \frac{1}{\omega^{j}} \geq \beta_{0}$, which leads to

$$
\beta_{s} \geq \frac{1}{\omega^{s}}\left(\beta_{0}-\frac{c_{0} \omega\left(\omega^{s}-1\right)}{(\omega-1) \omega^{s}}\right) \geq \beta_{0}\left(1-\frac{1}{m_{0}(\omega-1)}\right) \frac{1}{\omega^{s}} .
$$

Here, we use the fact that

$$
\rho_{0}:=\beta_{0}-\frac{c_{0} \omega\left(\omega^{s}-1\right)}{(\omega-1) \omega^{s}} \geq \beta_{0}-\frac{c_{0} \omega}{\omega-1}=\beta_{0}\left(1-\frac{1}{m_{0}(\omega-1)}\right)>0
$$

since $m_{0}>\frac{1}{\omega-1}$. This condition gives us a lower bound on $\beta_{s}$. 
Now, using [29], we have $\frac{\omega^{2} \beta_{s+1}^{2} m_{s+1}\left(m_{s+1}+3\right)}{\left(m_{s+1}+1\right)^{2}}=\beta_{s}^{2}$ and $\frac{\omega^{2}}{\left(m_{s+1}+1\right)^{2}} \leq \frac{1}{\left(m_{s}+1\right)^{2}}$ as in (51). Plugging these estimates into (59), we obtain

$$
\begin{array}{r}
\frac{4\|A\|^{2}}{\left(m_{s+1}+1\right)^{2}} d_{\mathcal{X}}\left(x^{\star}, \hat{x}^{K_{s+1}}\right)+\frac{\beta_{s+1}^{2} m_{s+1}\left(m_{s+1}+3\right)}{\left(m_{s+1}+1\right)^{2}} b_{\mathcal{Y}}\left(y^{\star}, \dot{y}^{s+1}\right) \leq \\
\frac{1}{\omega^{2}}\left[\frac{4\|A\|^{2}}{\left(m_{s}+1\right)^{2}} d_{\mathcal{X}}\left(x^{\star}, \hat{x}^{K_{s}}\right)+\frac{\beta_{s}^{2} m_{s}\left(m_{s}+3\right)}{\left(m_{s}+1\right)^{2}} b_{\mathcal{Y}}\left(y^{\star}, \dot{y}^{s}\right)\right] .
\end{array}
$$

By induction and using that $\hat{x}^{0}=\bar{x}^{0}$, we obtain

$$
\begin{aligned}
\frac{4\|A\|^{2}}{\left(m_{s}+1\right)^{2}} d_{\mathcal{X}}\left(x^{\star}, \hat{x}^{K_{s}}\right)+\frac{\beta_{s}^{2} m_{s}\left(m_{s}+3\right)}{\left(m_{s}+1\right)^{2}} b_{\mathcal{Y}}\left(y^{\star}, \dot{y}^{s}\right) & \leq \frac{1}{\omega^{2 s}}\left[\frac{4\|A\|^{2}}{\left(m_{0}+1\right)^{2}} d_{\mathcal{X}}\left(x^{\star}, \bar{x}^{0}\right)\right. \\
& \left.+\frac{\beta_{0}^{2} m_{0}\left(m_{0}+3\right)}{\left(m_{0}+1\right)^{2}} b_{\mathcal{Y}}\left(y^{\star}, \dot{y}^{0}\right)\right] .
\end{aligned}
$$

Since $m_{s}\left(m_{s}+3\right) \geq m_{s}-1$, combining 63 and (58), we obtain

$$
\begin{aligned}
S_{\beta_{s}}\left(\bar{x}^{K_{s+1}} ; \dot{y}^{s}\right) & \leq \frac{1}{\beta_{s} \omega^{2 s}}\left[\frac{4\|A\|^{2}}{\left(m_{0}+1\right)^{2}} d \mathcal{X}\left(x^{\star}, \bar{x}^{0}\right)+\frac{\beta_{0}^{2} m_{0}\left(m_{0}+3\right)}{\left(m_{0}+1\right)^{2}} b \mathcal{Y}\left(y^{\star}, \dot{y}^{0}\right)\right] \\
& \leq \frac{R_{0}^{2}}{\beta_{s} \omega^{2 s}}
\end{aligned}
$$

where $R_{0}:=\left[\frac{4\|A\|^{2}}{\left(m_{0}+1\right)^{2}} d_{\mathcal{X}}\left(x^{\star}, \bar{x}^{0}\right)+\frac{\beta_{0}^{2} m_{0}\left(m_{0}+3\right)}{\left(m_{0}+1\right)^{2}} b_{\mathcal{Y}}\left(y^{\star}, \dot{y}^{0}\right)\right]^{1 / 2}$.

Using (60) and (61) of $\beta_{s}$ and $m_{s}$ into (64), we obtain

$$
S_{\beta_{s}}\left(\bar{x}^{K_{s+1}} ; \dot{y}^{s}\right) \leq \frac{R_{0}^{2}}{\rho_{0} \omega^{s}} \leq \frac{\omega \kappa_{0} R_{0}^{2}}{\rho_{0}\left[(\omega-1) K_{s+1}+\kappa_{0}\right]} .
$$

Here, we use the same argument as in Theorem 3.1 to bound $\omega^{s}$ via the number of iterations $K_{s+1}$ as $\omega^{s} \geq \frac{(\omega-1) K_{s+1}+\kappa_{0}}{\omega \kappa_{0}}$, and $\rho_{0}:=\beta_{0}\left(1-\frac{1}{m_{0}(\omega-1)}\right)>0$.

Our next step is using 63 to bound $\left\|\dot{y}^{s}-y^{\star}\right\|_{\mathcal{Y}}$. Clearly, $\frac{\beta_{s}^{2} m_{s}\left(m_{s}+3\right)}{\left(m_{s}+1\right)^{2}}=$ $\frac{\beta_{s-1}^{2}}{\omega^{2}} \geq \frac{\rho_{0}^{2}}{\omega^{2 s}}$ by 61 . Using 63 , and strong convexity of $b_{\mathcal{Y}}$ with respect to the given norm, we can show that

$$
\frac{1}{2}\left\|\dot{y}^{s}-y^{\star}\right\|_{\mathcal{Y}}^{2} \leq b_{\mathcal{Y}}\left(y^{\star}, \dot{y}^{s}\right) \leq \frac{R_{0}^{2}}{\rho_{0}^{2}} .
$$

6.2. a The first estimate of 30 .

First, using Lemma 3.1 and by defining $\bar{\beta}_{s}:=\beta_{s} L_{b y}$ we write

$$
\begin{aligned}
f\left(\bar{x}^{K_{s+1}}\right)- & f^{\star} \geq \bar{\beta}_{s}\left\langle\dot{y}^{s}, y^{\star}\right\rangle-\left\|y^{\star}\right\| \operatorname{dist}_{\mathcal{K}}\left(A \bar{x}^{K_{s+1}}-b+\bar{\beta}_{s} \dot{y}^{s}\right) \\
& \geq \bar{\beta}_{s}\left\langle\dot{y}^{s}, y^{\star}\right\rangle-\left\|y^{\star}\right\| \operatorname{dist}_{\mathcal{K}}\left(A \bar{x}^{K_{s+1}}-b\right)-\left\|y^{\star}\right\| \bar{\beta}_{s}\left(\left\|\dot{y}^{s}-y^{\star}\right\|+\left\|y^{\star}\right\|\right) \\
\geq & -2 \bar{\beta}_{s}\left\|y^{\star}\right\|\left\|\dot{y}^{s}-y^{\star}\right\|-\left\|y^{\star}\right\| \operatorname{dist}_{\mathcal{K}}\left(A \bar{x}^{K_{s+1}}-b\right)
\end{aligned}
$$

By using the bound of $\left\|\dot{y}^{s}-y^{\star}\right\|$ from 66 along with $\beta_{s} \leq \frac{\beta_{0}}{\omega_{s}} \leq \frac{\omega \beta_{0} \kappa_{0}}{(\omega-1) K_{s+1}+\kappa_{0}}$, we conclude that

$$
f\left(\bar{x}^{K_{s+1}}\right)-f^{\star} \geq-\left\|y^{\star}\right\| \operatorname{dist}_{\mathcal{K}}\left(A \bar{x}^{K_{s+1}}-b\right)-\frac{2 \sqrt{2} \omega \beta_{0} L_{b_{\mathcal{Y}}} \kappa_{0}\left\|y^{\star}\right\| R_{0}}{\rho_{0}\left[(\omega-1) K_{s+1}+\kappa_{0}\right]} .
$$


6.2.b The second estimate of 30 .

Using Lemma 3.1, we have

$$
\begin{aligned}
f\left(\bar{x}^{K_{s+1}}\right)-f^{\star} & \leq S_{\beta_{s}}\left(\bar{x}^{K_{s+1}} ; \dot{y}^{s}\right)+\frac{\bar{\beta}_{s}}{2}\left\|\dot{y}^{s}\right\|^{2} \\
& \leq S_{\beta_{s}}\left(\bar{x}^{K_{s+1}} ; \dot{y}^{s}\right)+\frac{\bar{\beta}_{s}}{2}\left(\left\|\dot{y}^{s}-y^{\star}\right\|^{2}+\left\|y^{\star}\right\|^{2}\right) .
\end{aligned}
$$

Combining this bound, (65) and $(66)$ into 68 gives the second bound of $(30)$.

6.2.c Third estimate of 30 .

Finally, we note that, by using (61) and 64), we can bound $\frac{2 S_{\beta_{s}}\left(\bar{x}^{K_{s+1}} ; \dot{y}^{s}\right)}{\beta_{s}} \leq$ $\frac{2 R_{0}^{2}}{\rho_{0}^{2}}$. Using this upper bound, (27) and 66 into the third estimate of $(26)$, we obtain the third bound of 30 .

\section{Appendix: The proof of technical results}

This appendix provides the missing proof of the results in the main text.

\subsection{The proof of Example 3.1}

In this example, we have $b y(y, \dot{y})=\frac{1}{2}\|y-\dot{y}\|^{2}$. First, from the definition 22 of $g_{\beta}(A x ; \dot{y})$, by using the definition of $s_{\mathcal{K}}$, we write

$$
\begin{aligned}
g_{\beta}(A x ; \dot{y}) & =\min _{u \in \mathcal{K}} \max _{y \in \mathbb{R}^{n}}\{\langle A x-b-u, y\rangle-\beta b \mathcal{Y}(y, \dot{y})\} \\
& =\min _{u \in \mathcal{K}} \max _{y \in \mathbb{R}^{n}}\left\{\langle A x-b-u, y\rangle-\frac{\beta}{2}\|y-\dot{y}\|^{2}\right\} .
\end{aligned}
$$

The optimality condition of the max problem on the right hand side of the previous inequality is $A x-b-u-\beta(y-\dot{y})=0$, which implies $y=\dot{y}+\frac{1}{\beta}(A x-b-$ $u)$. In this case, $\langle A x-b-u, y\rangle-\frac{\beta}{2}\|y-\dot{y}\|^{2}=\frac{1}{2 \beta}\|A x-b-u\|^{2}+\langle\dot{y}, A x-b-u\rangle=$ $\frac{1}{2 \beta}\|A x-b-u+\beta \dot{y}\|^{2}-\frac{\beta}{2}\|\dot{y}\|^{2}$. Hence, we obtain

$$
\begin{aligned}
g_{\beta}(A x ; \dot{y}) & =\min _{u \in \mathcal{K}}\left\{\frac{1}{2 \beta}\|u-(A x-b+\beta \dot{y})\|^{2}\right\}-\frac{\beta}{2}\|\dot{y}\|^{2} \\
& =\frac{1}{2 \beta} \operatorname{dist}_{\mathcal{K}}(A x-b+\beta \dot{y})^{2}-\frac{\beta}{2}\|\dot{y}\|^{2},
\end{aligned}
$$

which is (23). In addition, this implies $u=\operatorname{proj}_{\mathcal{K}}(A x-b+\beta \dot{y})$. Hence, we obtain $y_{\beta}^{*}(A x ; \dot{y})=\dot{y}+\frac{1}{\beta}(A x-b-u)=\dot{y}+\frac{1}{\beta}\left(A x-b-\operatorname{proj}_{\mathcal{K}}(A x-b+\beta \dot{y})\right)$, which is exactly (24).

If $\mathcal{K}$ is a cone, then using Moreau's decomposition [2, Theorem 6.30], we can show that

$$
A x-b+\beta \dot{y}-\operatorname{proj}_{\mathcal{K}}(A x-b+\beta \dot{y})=\operatorname{proj}_{\mathcal{K}^{\circ}}(A x-b+\beta \dot{y}),
$$

where $K^{\circ}$ is the polar set of $\mathcal{K}$. Since $\mathcal{K}$ is a cone, $\mathcal{K}^{\circ}=-\mathcal{K}^{*}$, where $\mathcal{K}^{*}$ is the dual cone of $\mathcal{K}$. Hence, we have $y_{\beta}^{*}(A x ; \dot{y})=\operatorname{proj}_{-\mathcal{K}^{*}}\left(\dot{y}+\frac{1}{\beta}(A x-b)\right)$. 
7.2 The proof of Lemma 3.1: Optimality bounds for (3).

Using the property of distance function, we can derive

$$
\begin{aligned}
\operatorname{dist}_{\mathcal{K}}(A \bar{x}-b) & \leq \operatorname{dist}_{\mathcal{K}}(A \bar{x}-b+\beta \dot{y})+\operatorname{dist}_{\mathcal{K}}(\beta \dot{y}) \\
& =\operatorname{dist}_{\mathcal{K}}(A \bar{x}-b+\beta \dot{y})+\min _{u \in \mathcal{K}}\|u-\beta \dot{y}\| \\
& \stackrel{(i)}{\leq} \operatorname{dist}_{\mathcal{K}}(A \bar{x}-b+\beta \dot{y})+\beta\|\dot{y}\| \\
& \leq \operatorname{dist}_{\mathcal{K}}(A \bar{x}-b+\beta \dot{y})+\beta\left(\left\|\dot{y}-y^{\star}\right\|+\left\|y^{\star}\right\|\right)
\end{aligned}
$$

where $(i)$ holds since $\mathbf{0}^{n} \in \mathcal{K}$. Similarly, we can start from $\operatorname{dist}_{\mathcal{K}}(A \bar{x}-b+\beta \dot{y}) \leq$ $\operatorname{dist}_{\mathcal{K}}(A \bar{x}-b)+\operatorname{dist}_{\mathcal{K}}(\beta \dot{y})$, to get the similar bound

$$
\operatorname{dist}_{\mathcal{K}}(A \bar{x}-b+\beta \dot{y}) \leq \operatorname{dist}_{\mathcal{K}}(A \bar{x}-b)+\beta\left(\left\|\dot{y}-y^{\star}\right\|+\left\|y^{\star}\right\|\right) .
$$

Let $y^{\star}$ be an arbitrary optimal solution of the dual problem (6). Applying the strong duality condition for (3) and (6), we have

$$
\begin{aligned}
-f\left(x^{\star}\right) & =-f^{\star}=D^{\star}=f^{*}\left(-A^{T} y^{\star}\right)+\left\langle b, y^{\star}\right\rangle+s_{\mathcal{K}}\left(y^{\star}\right) \\
& \stackrel{(i)}{\geq}\left\langle b-A \bar{x}, y^{\star}\right\rangle-f(\bar{x})+s_{\mathcal{K}}\left(y^{\star}\right) \stackrel{(i i)}{=} \max _{u \in \mathcal{K}}\left\{\left\langle y^{\star}, b-A \bar{x}+u\right\rangle\right\}-f(\bar{x}) \\
& =\max _{u \in \mathcal{K}}\left\{-\left\langle y^{\star}, A \bar{x}-b-u\right\rangle\right\}-f(\bar{x}),
\end{aligned}
$$

where $(i)$ follows by the definition of conjugate function $f^{*}(\cdot)$, and $(i i)$ follows by the definition of support function $s_{\mathcal{K}}(\cdot)$. By rearranging, we get the following relation:

$$
f(\bar{x})-f\left(x^{\star}\right) \geq \max _{u \in \mathcal{K}}\left\{-\left\langle y^{\star}, A \bar{x}-b-u\right\rangle\right\} .
$$

Now, since $\left\langle y^{\star}, A \bar{x}-b-u+\beta \dot{y}\right\rangle \leq\left\|y^{\star}\right\|\|A \bar{x}-b-u+\beta \dot{y}\|$, we have

$$
\begin{aligned}
\max _{u \in \mathcal{K}}\left\{-\left\langle y^{\star}, A \bar{x}-b-u\right\rangle\right\}-\beta\left\langle y^{\star}, \dot{y}\right\rangle & \geq \max _{u \in \mathcal{K}}\left\{-\left\|y^{\star}\right\|\|A \bar{x}-b-u+\beta \dot{y}\|\right\} \\
& =-\left\|y^{\star}\right\| \min _{u \in \mathcal{K}}\|A \bar{x}-b+\beta \dot{y}-u\| \\
& =-\left\|y^{\star}\right\| \operatorname{dist}_{\mathcal{K}}(A \bar{x}-b+\beta \dot{y}) .
\end{aligned}
$$

Combining the above two inequalities, we obtain

$$
f(\bar{x})-f\left(x^{\star}\right) \geq \beta\left\langle y^{\star}, \dot{y}\right\rangle-\left\|y^{\star}\right\| \operatorname{dist}_{\mathcal{K}}(A \bar{x}-b+\beta \dot{y}),
$$

which is the first estimate of 26 .

Since we assume $b_{\mathcal{Y}}(y, \dot{y})$ with Lipschitz gradients, we can write

$$
\begin{aligned}
g_{\beta}(A x ; \dot{y}) & =\min _{u \in \mathcal{K}} \max _{y \in \mathbb{R}^{n}}\left\{\langle A x-b-u, y\rangle-\beta b_{\mathcal{Y}}(y, \dot{y})\right\} \\
& \stackrel{(i i)}{\geq} \min _{u \in \mathcal{K}} \max _{y \in \mathbb{R}^{n}}\left\{\langle A x-b-u, y\rangle-\frac{\beta L_{b \mathcal{Y}}}{2}\|y-\dot{y}\|^{2}\right\} \\
& \stackrel{(i i)}{=} \frac{1}{2 \beta_{b}} \operatorname{dist}_{\mathcal{K}}\left(A x-b+\beta_{b} \dot{y}\right)^{2}-\frac{\beta_{b}}{2}\|\dot{y}\|^{2},
\end{aligned}
$$

where $(i)$ holds because of the Lipschitz gradient assumption on $b \mathcal{y}$, and $(i i)$ follows from Example 3.1 and defining $\beta_{b}:=\beta L_{b y}$. 
Invoking (71) with $\beta=\beta_{b}$ and combining it with 72 and $S_{\beta}(\cdot ; \dot{y})=$ $f(\bar{x})+g_{\beta}(\bar{x} ; \dot{y})-f\left(x^{\star}\right)$ gives

$$
\beta_{b}\left\langle y^{\star}, \dot{y}\right\rangle-\left\|y^{\star}\right\| \operatorname{dist}_{\mathcal{K}}\left(A \bar{x}-b+\beta_{b} \dot{y}\right) \leq f(\bar{x})-f\left(x^{\star}\right)=S_{\beta}(\bar{x} ; \dot{y})-g_{\beta}(A \bar{x} ; \dot{y}) .
$$

Using 73 into this inequalty, we have

$$
\begin{aligned}
\beta_{b}\left\langle y^{\star}, \dot{y}\right\rangle-\left\|y^{\star}\right\| \operatorname{dist}_{\mathcal{K}}\left(A \bar{x}-b+\beta_{b} \dot{y}\right) & \leq S_{\beta}(\bar{x} ; \dot{y})-\frac{1}{2 \beta_{b}} \operatorname{dist}_{\mathcal{K}}\left(A x-b+\beta_{b} \dot{y}\right)^{2} \\
& +\frac{\beta_{b}}{2}\|\dot{y}\|^{2} .
\end{aligned}
$$

Let $t:=\operatorname{dist}_{\mathcal{K}}\left(A \bar{x}-b+\beta_{b} \dot{y}\right)$. Then, this inequality becomes

$$
\frac{1}{2 \beta_{b}} t^{2}-\left\|y^{\star}\right\| t-\left(S_{\beta}(\bar{x} ; \dot{y})+\frac{\beta_{b}}{2}\|\dot{y}\|^{2}-\beta_{b}\left\langle y^{\star}, \dot{y}\right\rangle\right) \leq 0 .
$$

By using the strong convexity of $b_{y}(\cdot, \dot{y})$ with respect to the corresponding norm, one can plug in the optimality condition of the maximization problem 10 to derive

$$
2 b_{\mathcal{Y}}\left(y^{\star}, \dot{y}\right)+\frac{2}{\beta} S_{\beta}(\bar{x} ; \dot{y}) \geq\left\|y_{\beta}^{*}(A \bar{x} ; \dot{y})-\dot{y}\right\|^{2} \geq 0 .
$$

By using this inequality and strong convexity of $b_{\mathcal{Y}}(\cdot, \dot{y})$, we conclude that $\| y^{\star}-$ $\dot{y} \|^{2}+\frac{2}{\beta} S_{\beta}(\bar{x} ; \dot{y}) \geq 0$, therefore the inequation 75 has solution. Consequently, we can write that

$$
t^{*}=\operatorname{dist}_{\mathcal{K}}\left(A \bar{x}-b+\beta_{b} \dot{y}\right) \leq \beta_{b}\left[\left\|y^{\star}\right\|+\left(\left\|y^{\star}-\dot{y}\right\|^{2}+\frac{2}{\beta_{b}} S_{\beta}(\bar{x} ; \dot{y})\right)^{1 / 2}\right],
$$

which is the third estimate of (26).

Finally, plugging (73) into (74), we obtain the second estimate of $(26)$.

\section{References}

1. Sanjeev Arora, Mikhail Khodak, Nikunj Saunshi, and Kiran Vodrahalli. A compressed sensing view of unsupervised text embeddings, bag-of-n-grams, and LSTMs. In International Conference on Learning Representations, 2018.

2. H.H. Bauschke and P. Combettes. Convex analysis and monotone operators theory in Hilbert spaces. Springer-Verlag, 2011.

3. A. Beck and M. Teboulle. A fast iterative shrinkage-thresholding agorithm for linear inverse problems. SIAM J. Imaging Sci., 2(1):183-202, 2009.

4. A. Beck and M. Teboulle. Smoothing and first order methods: A unified framework. SIAM J. Optim., 22(2):557-580, 2012.

5. Dimitri P. Bertsekas. Constrained Optimization and Lagrange Multiplier Methods. Athena Scientific, 1996.

6. Allan Borodin, Ran El-Yaniv, and Vincent Gogan. Can we learn to beat the best stock. In Advances in Neural Information Processing Systems, pages 345-352, 2004.

7. Jonathan M Borwein, Jon D Vanderwerff, et al. Convex functions: constructions, characterizations and counterexamples, volume 109. Cambridge University Press Cambridge, 2010.

8. Radu Ioan Bot and Christopher Hendrich. A variable smoothing algorithm for solving convex optimization problems. TOP, 23(1):124-150, 2012. 
9. R.I. Bot and C. Hendrich. A double smoothing technique for solving unconstrained nondifferentiable convex optimization problems. Compt. Optim. Appl., 54(2):239-262, 2013.

10. S. Boyd, N. Parikh, E. Chu, B. Peleato, and J. Eckstein. Distributed optimization and statistical learning via the alternating direction method of multipliers. Foundations and Trends in Machine Learning, 3(1):1-122, 2011.

11. Joshua Brodie, Ingrid Daubechies, Christine De Mol, Domenico Giannone, and Ignace Loris. Sparse and stable markowitz portfolios. Proceedings of the National Academy of Sciences, 106(30):12267-12272, 2009.

12. Emmanuel J Candès, Justin Romberg, and Terence Tao. Robust uncertainty principles: Exact signal reconstruction from highly incomplete frequency information. IEEE Transactions on information theory, 52(2):489-509, 2006.

13. A. Chambolle and T. Pock. A first-order primal-dual algorithm for convex problems with applications to imaging. J. Math. Imaging Vis., 40(1):120-145, 2011.

14. A. Chambolle and T. Pock. On the ergodic convergence rates of a first-order primal-dual algorithm. Math. Program., 159(1-2):253-287, 2016.

15. Chih-Chung Chang and Chih-Jen Lin. LIBSVM: A library for support vector machines. ACM Transactions on Intelligent Systems and Technology, 2:27:1-27:27, 2011.

16. G. Chen and M. Teboulle. Convergence analysis of a proximal-like minimization algorithm using Bregman functions. SIAM J. Optim., 3(3):538-543, 1993.

17. Scott Shaobing Chen, David L Donoho, and Michael A Saunders. Atomic decomposition by basis pursuit. SIAM review, 43(1):129-159, 2001.

18. P. Combettes and Pesquet J.-C. Signal recovery by proximal forward-backward splitting. In Fixed-Point Algorithms for Inverse Problems in Science and Engineering, pages 185212. Springer-Verlag, 2011.

19. Laurent Condat. A primal-dual splitting method for convex optimization involving lipschitzian, proximable and linear composite terms. Journal of Optimization Theory and Applications, 158(2):460-479, 2013.

20. D. Davis. Convergence rate analysis of the forward-Douglas-Rachford splitting scheme. SIAM J. Optim., 25(3):1760-1786, 2015.

21. D. Davis and W. Yin. Faster convergence rates of relaxed Peaceman-Rachford and ADMM under regularity assumptions. Math. Oper. Res., 2014.

22. O. Devolder, F. Glineur, and Y. Nesterov. Double smoothing technique for large-scale linearly constrained convex optimization. SIAM J. Optim., 22(2):702-727, 2012.

23. David L Donoho. Compressed sensing. IEEE Transactions on information theory, 52(4):1289-1306, 2006.

24. J. Eckstein. Nonlinear proximal point algorithms using Bregman functions, with applications to convex programming. Math. Oper. Res., 18(1):202-226, 1993.

25. Ehsan Elhamifar and René Vidal. Sparse subspace clustering. In Computer Vision and Pattern Recognition, 2009. CVPR 2009. IEEE Conference on, pages 2790-2797. IEEE, 2009.

26. Ehsan Elhamifar and Rene Vidal. Sparse subspace clustering: Algorithm, theory, and applications. IEEE transactions on pattern analysis and machine intelligence, 35(11):2765-2781, 2013.

27. J. E. Esser. Primal-dual algorithm for convex models and applications to image restoration, registration and nonlocal inpainting. $\mathrm{PhD}$ Thesis, University of California, Los Angeles, Los Angeles, USA, 2010.

28. O. Fercoq and Z. Qu. Restarting accelerated gradient methods with a rough strong convexity estimate. arXiv preprint arXiv:1609.07358, 2016.

29. Xiang Gao and Shu-Zhong Zhang. First-order algorithms for convex optimization with nonseparable objective and coupled constraints. Journal of the Operations Research Society of China, 5(2):131-159, 2017.

30. Athinodoros S. Georghiades, Peter N. Belhumeur, and David J. Kriegman. From few to many: Illumination cone models for face recognition under variable lighting and pose. IEEE transactions on pattern analysis and machine intelligence, 23(6):643-660, 2001.

31. P. Giselsson and S. Boyd. Monotonicity and Restart in Fast Gradient Methods. In IEEE Conference on Decision and Control, pages 5058-5063, Los Angeles, USA, December 2014. CDC. 
32. B.S. He and X.M. Yuan. On the $O(1 / n)$ convergence rate of the Douglas-Rachford alternating direction method. SIAM J. Numer. Anal., 50:700-709, 2012.

33. K. C. Kiwiel. Proximal minimization methods with generalized Bregman functions SIAM J. Control Optim., 35(4):1142-1168, 1997.

34. Guanghui Lan, Zhaosong Lu, and Renato DC Monteiro. Primal-dual first-order methods with iteration-complexity for cone programming. Mathematical Programming, 126(1):129, 2011.

35. Guanghui Lan and Renato DC Monteiro. Iteration-complexity of first-order penalty methods for convex programming. Mathematical Programming, 138(1-2):115-139, 2013.

36. Guanghui Lan and Renato DC Monteiro. Iteration-complexity of first-order augmented lagrangian methods for convex programming. Mathematical Programming, 155(1-2):511-547, 2016.

37. H. Li and Z. Lin. Accelerated Alternating Direction Method of Multipliers: an Optimal $\mathcal{O}(1 / k)$ Nonergodic Analysis. arXiv preprint arXiv:1608.06366, 2016.

38. Andrew L. Maas, Raymond E. Daly, Peter T. Pham, Dan Huang, Andrew Y. Ng, and Christopher Potts. Learning word vectors for sentiment analysis. In Proceedings of the 49th Annual Meeting of the Association for Computational Linguistics: Human Language Technologies, pages 142-150, Portland, Oregon, USA, June 2011. Association for Computational Linguistics.

39. R.D.C. Monteiro and B.F. Svaiter. Iteration-complexity of block-decomposition algorithms and the alternating direction method of multipliers. SIAM J. Optim., 23(1):475$507,2013$.

40. R.D.C. Monteiro and B.F. Svaiter. Iteration-complexity of block-decomposition algorithms and the alternating minimization augmented Lagrangian method. SIAM J. Optim., 23(1):475-507, 2013.

41. I. Necoara and J.A.K. Suykens. Applications of a smoothing technique to decomposition in convex optimization. IEEE Trans. Automatic control, 53(11):2674-2679, 2008.

42. Ion Necoara, Andrei Patrascu, and Francois Glineur. Complexity certifications of first order inexact lagrangian and penalty methods for conic convex programming. arXiv preprint arXiv:1506.05320, 2015.

43. Valentin Nedelcu, Ion Necoara, and Quoc Tran-Dinh. Computational complexity of inexact gradient augmented lagrangian methods: application to constrained mpc. SIAM Journal on Control and Optimization, 52(5):3109-3134, 2014.

44. A. Nemirovskii and D. Yudin. Problem Complexity and Method Efficiency in Optimization. Wiley Interscience, 1983.

45. Y. Nesterov. Introductory lectures on convex optimization: A basic course, volume 87 of Applied Optimization. Kluwer Academic Publishers, 2004.

46. Y. Nesterov. Excessive gap technique in nonsmooth convex minimization. SIAM J. Optim., 16(1):235-249, 2005.

47. Y. Nesterov. Smooth minimization of non-smooth functions. Math. Program., 103(1):127-152, 2005.

48. B. O'Donoghue and E. Candes. Adaptive Restart for Accelerated Gradient Schemes. Found. Comput. Math., 15:715-732, 2015.

49. Y. Ouyang, Y. Chen, G. Lan, and E. JR. Pasiliao. An accelerated linearized alternating direction method of multiplier. SIAM J. Imaging Sci., 8(1):644-681, 2015.

50. Yuyuan Ouyang, Yunmei Chen, Guanghui Lan, and Eduardo Pasiliao Jr. An accelerated linearized alternating direction method of multipliers. SIAM Journal on Imaging Sciences, 8(1):644-681, 2015.

51. N. Parikh and S. Boyd. Proximal algorithms. Foundations and Trends in Optimization, 1(3):123-231, 2013

52. Jeffrey Pennington, Richard Socher, and Christopher D. Manning. Glove: Global vectors for word representation. In Empirical Methods in Natural Language Processing (EMNLP), pages 1532-1543, 2014.

53. Farhad Pourkamali-Anaraki and Stephen Becker. Efficient solvers for sparse subspace clustering. arXiv preprint arXiv:1804.06291, 2018.

54. James Renegar. Efficient first-order methods for linear programming and semidefinite programming. arXiv preprint arXiv:1409.5832, 2014. 
55. R. Shefi and M. Teboulle. Rate of Convergence Analysis of Decomposition Methods Based on the Proximal Method of Multipliers for Convex Minimization. SIAM J. Optim., 24(1):269-297, 2014.

56. W. Su, S. Boyd, and E. Candes. A differential equation for modeling Nesterov's accelerated gradient method: Theory and insights. In Advances in Neural Information Processing Systems (NIPS), pages 2510-2518, 2014.

57. Q. Tran-Dinh. Proximal Alternating Penalty Algorithms for Constrained Convex Optimization. Working Paper (STOR-UNC-Chapel Hill), Preprint: arXiv:1711.01367 pages 1-36, 2017.

58. Q. Tran-Dinh, O. Fercoq, and V. Cevher. A smooth primal-dual optimization framework for nonsmooth composite convex minimization. Accepted for publication on SIAM J. Optim., pages 1-29, 2017.

59. P. Tseng. On accelerated proximal gradient methods for convex-concave optimization. Submitted to SIAM J. Optim, 2008.

60. Quang Van Nguyen, Olivier Fercoq, and Volkan Cevher. Smoothing technique for nonsmooth composite minimization with linear operator. arXiv preprint arXiv:1706.05837 2017.

61. C. B. Vũ. A splitting algorithm for dual monotone inclusions involving cocoercive operators. Advances in Computational Mathematics, 38(3):667-681, 2013.

62. Hansheng Wang, Guodong $\mathrm{Li}$, and Guohua Jiang. Robust regression shrinkage and consistent variable selection through the lad-lasso. Journal of Business 86 Economic Statistics, 25(3):347-355, 2007.

63. Sinong Wang and Ness Shroff. A new alternating direction method for linear programming. In Advances in Neural Information Processing Systems, pages 1479-1487, 2017.

64. Lyndon White, Roberto Togneri, Wei Liu, and Mohammed Bennamoun. Generating bags of words from the sums of their word embeddings. In 17th International Conference on Intelligent Text Processing and Computational Linguistics (CICLing), 2016.

65. B. E. Woodworth and N. Srebro. Tight complexity bounds for optimizing composite objectives. In Advances in neural information processing systems (NIPS), pages 3639$3647,2016$.

66. Stephen J Wright. Optimization algorithms for data analysis.

67. Yangyang $\mathrm{Xu}$. Accelerated first-order primal-dual proximal methods for linearly constrained composite convex programming. SIAM Journal on Optimization, 27(3):14591484, 2017.

68. Yangyang $\mathrm{Xu}$. Iteration complexity of inexact augmented lagrangian methods for constrained convex programming. arXiv preprint arXiv:1711.05812u2, 2017.

69. Yi Xu, Yan Yan, Qihang Lin, and Tianbao Yang. Homotopy smoothing for non-smooth problems with lower complexity than $o(1 / \epsilon)$. In Advances in Neural Information Processing Systems, pages 1208-1216, 2016.

70. Ji Zhu, Saharon Rosset, Robert Tibshirani, and Trevor J Hastie. 1-norm support vector machines. In Advances in neural information processing systems, pages 49-56, 2004. 